\title{
A MULTIVARIATE FIT LUMINOSITY FUNCTION AND WORLD MODEL FOR LONG GAMMA-RAY BURSTS
}

\author{
AMIR SHAHMORADI \\ Institute for Fusion Studies, The University of Texas at Austin, TX 78712, USA; amir@ physics.utexas.edu \\ Received 2012 September 5; accepted 2013 January 17; published 2013 March 15
}

\begin{abstract}
It is proposed that the luminosity function, the rest-frame spectral correlations, and distributions of cosmological long-duration (Type-II) gamma-ray bursts (LGRBs) may be very well described as a multivariate log-normal distribution. This result is based on careful selection, analysis, and modeling of LGRBs' temporal and spectral variables in the largest catalog of GRBs available to date: 2130 BATSE GRBs, while taking into account the detection threshold and possible selection effects. Constraints on the joint rest-frame distribution of the isotropic peak luminosity $\left(L_{\text {iso }}\right)$, total isotropic emission $\left(E_{\text {iso }}\right)$, the time-integrated spectral peak energy $\left(E_{p, z}\right)$, and duration $\left(T_{90, z}\right)$ of LGRBs are derived. The presented analysis provides evidence for a relatively large fraction of LGRBs that have been missed by the BATSE detector with $E_{\text {iso }}$ extending down to $\sim 10^{49} \mathrm{erg}$ and observed spectral peak energies $\left(E_{p}\right)$ as low as $\sim 5 \mathrm{keV}$. LGRBs with rest-frame duration $T_{90, z} \lesssim 1 \mathrm{~s}$ or observer-frame duration $T_{90} \lesssim 2 \mathrm{~s}$ appear to be rare events $(\lesssim 0.1 \%$ chance of occurrence). The model predicts a fairly strong but highly significant correlation ( $\rho=0.58 \pm 0.04)$ between $E_{\text {iso }}$ and $E_{p, z}$ of LGRBs. Also predicted are strong correlations of $L_{\text {iso }}$ and $E_{\text {iso }}$ with $T_{90, z}$ and moderate correlation between $L_{\text {iso }}$ and $E_{p, z}$. The strength and significance of the correlations found encourage the search for underlying mechanisms, though undermine their capabilities as probes of dark energy's equation of state at high redshifts. The presented analysis favors-but does not necessitate - a cosmic rate for BATSE LGRBs tracing metallicity evolution consistent with a cutoff $Z / Z_{\odot} \sim 0.2-0.5$, assuming no luminosity-redshift evolution.
\end{abstract}

Key words: dark energy - gamma-ray burst: general - methods: statistical

Online-only material: color figures

\section{INTRODUCTION}

Ever since the discovery of the first gamma-ray burst (GRB) by the Vela satellites in 1967 (Klebesadel et al. 1973), there has been tremendous effort and attempts to constrain the energetics, luminosity function (LF), and the underlying mechanism responsible for these events. Early observations by the Konus (Mazets \& Golenetskii 1981) and Ginga (Fenimore et al. 1988; Nishimura 1988) gamma/X-ray instruments suggested a possible link between GRBs and neutron stars with output energy ranges of the order of $\sim 10^{40} \mathrm{erg}$. With the launch of the Compton Gamma-Ray Observatory (CGRO), the Burst And Transient Source Experiment (BATSE) on board CGRO dramatically changed the understanding of GRBs. While previous catalogs (e.g., Atteia et al. 1987) indicated an isotropic distribution of GRB sources, the BATSE observations extended this isotropy down to the weakest bursts. The non-homogenous (e.g., Fenimore et al. 1993) and isotropic spacial event distribution (e.g., Meegan et al. 1992; Briggs 1993; Fishman et al. 1994) provided, for the first time, strong support for a cosmological versus galactic origin of GRBs, undermining neutron stars in the local universe as the potential candidates for some-if not all-classes of gamma-ray events. Furthermore, the joint duration-hardness distribution of GRBs provided a direct evidence for at least two separate classes of GRBs: long-soft versus short-hard (e.g., Kouveliotou et al. 1993, see also Figure 1 here).

The possibility of a cosmological origin for GRBs indicated an enormous output energy on the order of $\sim 10^{51} \mathrm{erg}$ (e.g., Dermer 1992). Nevertheless, an accurate description of the GRB LF also required knowledge of the GRB cosmic rate, information that could not be extracted from BATSE observations alone. This became possible only with the launch of the Italian-Dutch X-ray satellite BeppoSax (Boella et al. 1997) and the identi- fication of the first GRB with firmly measured cosmological redshift (Metzger et al. 1997) that marked the beginning of the afterglow era in the field of GRBs. The launch of the Swift satellite (Gehrels et al. 2004) was another milestone that revolutionized the study of GRBs by facilitating the X-ray afterglow observations (Burrows et al. 2005) and further ground-based follow-ups for redshift measurement.

Alongside the observational triumphs over a few decades, several theoretical models have stood up against the rivals based on the available evidence and GRB data. Most prominently, the Collapsar model (e.g., Woosley 1993) has been relatively successful in linking the long-duration class of gammaray bursts (LGRBs) to the final stages in the lives of massive stars, while the short-duration class of bursts (SGRBs) is generally attributed to the coalescence of compact binary systems (e.g., Paczynski 1986; Nakar 2007 and references therein). The two classes of SGRBs and LGRBs in this work correspond to Type-I and Type-II GRBs, respectively, according to the physical classification scheme of Zhang et al. (2007) and Bloom et al. (2008). Further refinement of the potential candidates, as the progenitors and the emission mechanism for both classes, requires more rigorous analysis of observational data in all possible energy frequencies. In particular, the prompt gamma-ray emission of LGRBs has been subject of intense observational and theoretical studies.

Beginning with BATSE observations, numerous authors have examined the prompt emission of LGRBs searching for potential underlying correlations among the spectral parameters (e.g., Nemiroff et al. 1994; Fenimore et al. 1995; Mallozzi et al. 1995; Petrosian \& Lee 1996; Brainerd 1997; Dezalay et al. 1997; Petrosian et al. 1999; Lloyd et al. 2000; Norris et al. 2005). The lack of known redshifts for BATSE events and poor knowledge of LGRB cosmic rates, however, strongly limited 
the prediction power of such analyses. Instead, the first direct evidence for potential correlations and constraints on the distributions of the prompt-emission spectral parameters came with a few LGRBs detected by BATSE, BeppoSax, IPN, or HETE-II satellites with measured redshifts (e.g., Reichart \& Lamb 2001; Amati et al. 2002; Ghirlanda et al. 2004; Yonetoku et al. 2004) and was further developed by the inclusion of Swift LGRBs (e.g., Schaefer 2007; Gehrels et al. 2009). Such findings, however, have been criticized for relying primarily on a handful of events with high signal-to-noise ratio $(\mathrm{S} / \mathrm{N})$ required for spectral analysis with afterglows sufficiently bright for redshift measurement, arguing that the proposed joint distributions of the spectral properties do not represent the entire underlying population of LGRBs (e.g., Band \& Preece 2005; Nakar \& Piran 2005; Li 2007; Butler et al. 2007, 2009; Shahmoradi \& Nemiroff 2009, 2010, 2011). Responding to criticisms, attempts were made to model the effects of different gamma-ray instruments' detection thresholds and the limiting effects of spectral analysis (e.g., Ghirlanda et al. 2008; Nava et al. 2008; see Shahmoradi \& Nemiroff 2011 for a review of relevant literature).

Despite significant progress, difficulties in modeling the complex effects of detector threshold on the multivariate distribution of the prompt-emission spectral properties and the lack of a sufficiently large sample of uniformly detected LGRBs has led the community to focus on individual spectral variables, most importantly the LF (e.g., Petrosian 1993; Schmidt 1999, 2001, 2009; Kommers et al. 2000; Kumar \& Piran 2000; Band 2001; Porciani \& Madau 2001; Sethi \& Bhargavi 2001; Stern et al. 2002; Guetta et al. 2005; Salvaterra \& Chincarini 2007; Salvaterra et al. 2009; Campisi et al. 2010; Wanderman \& Piran 2010; Salvaterra et al. 2012). Recently, Butler et al. (2010, hereafter B10) presented an elaborate multivariate analysis of Swift LGRBs, including the potential correlations among three temporal and spectral variables: total isotropic energy emission $\left(E_{\text {iso }}(\mathrm{erg})\right)$, a definition of duration $\left(T_{r 45}(\mathrm{~s})\right)$, and the spectral peak energy $\left(E_{p, z}(\mathrm{keV})\right)$ of the bursts. Focusing their analysis on the $E_{\mathrm{iso}}-E_{p, z}-T_{r 45}$ interrelation, B10 find a strong and significant correlation - but with a broad scatter-between the isotropic emission and the peak energy of the Swift LGRBs. Also realized by $\mathrm{B} 10$ is the possibility of a positive-and perhaps strong - correlation between the duration and the total energy output of the bursts. Moreover, to accommodate the potential existence of a large population of sub-luminous events, a broken power-law LF is used by B10.

It is known that the energy budget of GRBs must be limited and a turnover in the LF at the low-luminosity tail of the population is expected. However, the choice of the broken power law as the candidate LF is justified by the fact that current observational data cannot constrain this turnover point, which is expected to be far below the detection threshold of current gamma-ray instruments. The existence of a turnover point in the LF can have important clues for the underlying physics of LGRBs. The low-luminosity tail of the LF in such a case would be the result of the convolution of the stellar mass distribution with the LGRB rate as a function of the properties of massive dying stars, imposed by the mechanism.

Motivated by the search for the potential shape of the LGRB LF at low luminosity and the flurry of recent reports on the possible existence of correlations among LGRBs' temporal and spectral variables, the author of this paper has considered a wide variety of different statistical models that could incorporate and explain all observed correlations and spectral distributions, while preserving the prediction power of the model for the spectral properties of a potentially large fraction of LGRBs that could go undetected by current gamma-ray detectors. Here it will be shown that it is indeed possible to construct an LGRB world model capable of describing most (if not all) prompt-emission spectral and temporal properties observed in the current data catalogs. Toward this, the presented analysis is mainly focused on the largest catalog of GRBs available to date: the BATSE catalog of 2130 GRBs (Paciesas et al. 1999). The author shows that there is still an enormous amount of information buried in BATSE observations that need to be dug out by GRB researchers.

In the following sections, an example of such data mining on BATSE catalog will be presented: Section 2.1 presents an elaborate method for classifying GRBs into the two known subclasses of SGRBs and LGRBs. In Section 2.2 an LGRB world model capable of describing BATSE observations is presented, followed by a discussion of the procedure for fitting the model to data in Section 2.3. Univariate and multivariate goodness-of-fit $(\mathrm{GoF})$ tests are performed to ensure that the model predicts BATSE data accurately (Section 2.4). The results from model fitting and implications on the multivariate distribution of the prompt-emission spectral properties are discussed in Section 3. A summary of the analysis and important outcomes of the proposed LGRB world model will be presented in Section 4.

\section{GRB WORLD MODEL}

\subsection{Sample Selection}

Depending on their detection criteria, some gamma-ray detectors might facilitate detection of one class of bursts over the others. For example, the specific detector sensitivity of the Burst Alert Telescope (BAT) on board the Swift satellite (Gehrels et al. 2004) results in better detections of LGRBs over SGRBs (e.g., Band 2003, 2006). Therefore, a simple GRB classification method, such as a cutoff in the observed duration distributions of GRBs $\left(T_{90} \sim 3 \mathrm{~s}\right)$ generally results in LGRB samples that are minimally contaminated by SGRBs (e.g., B10). Compared to BAT, however, BATSE Large Area Detectors (LADs) had an increased relative sensitivity to SGRBs (e.g., Band 2003). Knowing that many temporal and spectral properties of LGRBs and SGRBs, most importantly $T_{90}$, overlap, identification of LGRBs in the BATSE catalog solely based on their $T_{90}$ will likely result in a significant number of misclassifications (e.g., Figure 1, center panel).

Thus, to ensure correct analysis of a long-duration class of GRBs, it is first necessary to collect the least biased sample of events, all belonging to LGRB category. The word "bias" here refers to the bias that might be introduced when using the traditional definition of GRB classes, based on a sharp cutoff on the duration variable $T_{90}$ (Kouveliotou et al. 1993), as it is generally assumed by many GRB researchers (e.g., Guetta et al. 2005; B10; Campisi et al. 2010; Wanderman \& Piran 2010) or not assumed or explicitly discussed (e.g., Salvaterra \& Chincarini 2007; Salvaterra et al. 2009, 2012). This goal is achieved by testing extensive varieties of classification and clustering methods, most importantly, the fuzzy clustering algorithms of Rousseeuw et al. (1996) and the method of fuzzy C-means (Dunn 1973; Bezdek 1981). Each BATSE-catalog GRB is assigned a probability (i.e., class coefficient) of belonging to the LGRB (versus SGRB) population according to the choice of clustering algorithm and the set of GRB variables used. This can be any combination of the peak flux $\left(P_{50-300}\right.$ photons s$\left.{ }^{-1} \mathrm{~cm}^{-2}\right)$ 

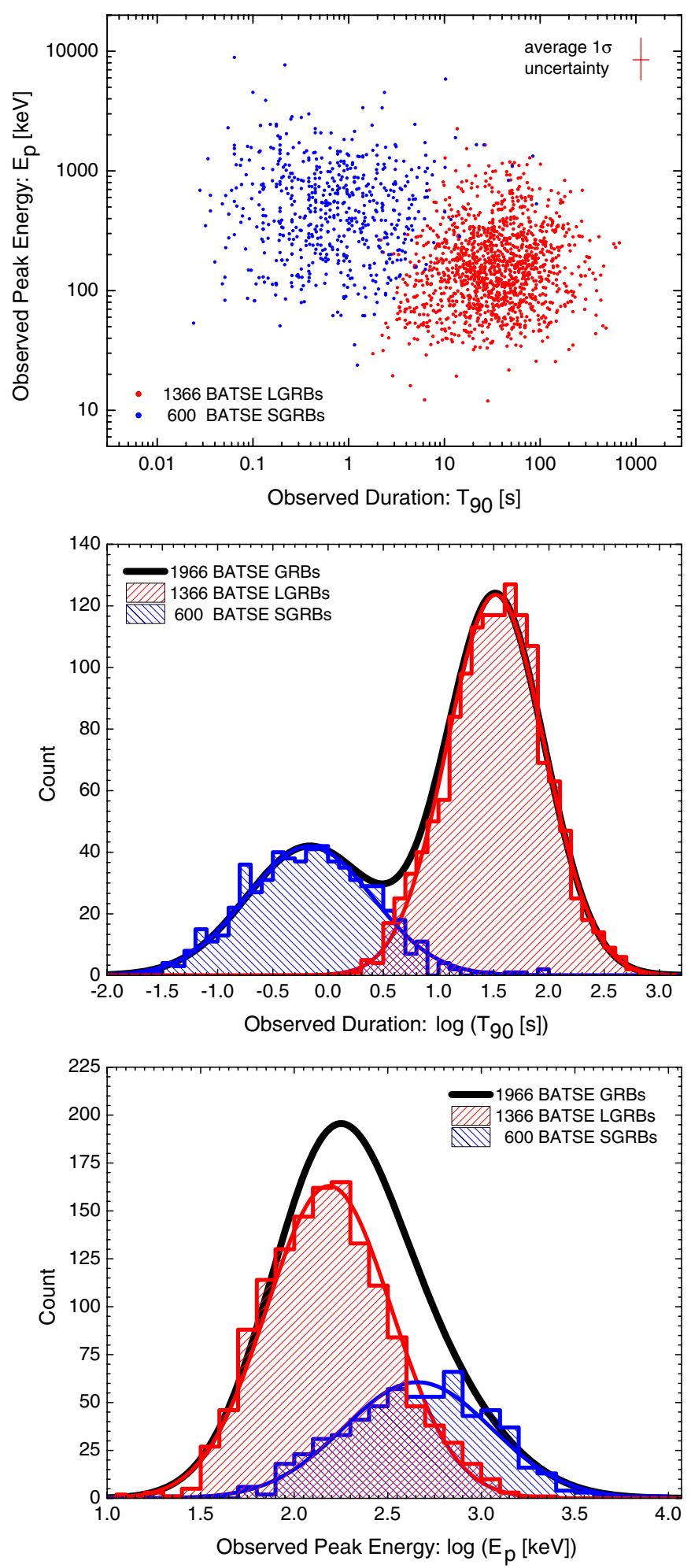

Figure 1. Classification of 1966 BATSE LGRBs according to the most suitable clustering algorithm and set of GRB variables (Section 2.1): fuzzy C-means classification on $E_{p}$ and $T_{90}$. Red and blue colors represent LGRB and SGRB classes, respectively, in all three plots. Top: the joint $T_{90}-E_{p}$ distribution. The uncertainties in LGRBs are derived from the empirical Bayes model discussed in Appendix C. Center: $T_{90}$ distribution. Bottom: $E_{p}$ distribution. $E_{p}$ estimates are taken from Shahmoradi \& Nemiroff (2010). Compare this plot to the plot of Figure 13 of Shahmoradi \& Nemiroff (2010), where the entire univariate $E_{p}$ distributions of BATSE GRBs were fit by a two-component Gaussian mixture.

(A color version of this figure is available in the online journal.) in the BATSE detection energy range, in three different timescales: 64, 256, and $1024 \mathrm{~ms}$; bolometric fluence

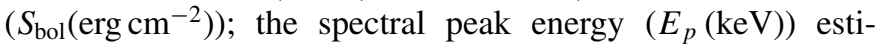
mates by Shahmoradi \& Nemiroff (2010); duration $\left(T_{90}(\mathrm{~s})\right)$; and the fluence-to-peak-flux ratio (FPR (s)). Then GRBs with LGRB class coefficient $>0.5$ are flagged as long-duration class bursts. Overall, the fuzzy C-means classification method with the two GRB variables $E_{p}$ and $T_{90}$ is preferred over other clustering methods and sets of GRB variables (cf. Appendix A). This leads to the selection of 1376 events as LGRBs out of the 1966 BATSE GRBs having measured temporal and spectral parameters mentioned above. ${ }^{1}$

As a further safety check to ensure minimal contamination of the sample by SGRBs, the light curves of 291 bursts among 1966 BATSE GRBs with LGRB class coefficients in the range of $0.3-0.7$ are visually inspected in the four main energy channels of BATSE LADs. This leads to reclassification of 17 events (originally flagged as LGRB by the clustering algorithm) to potentially SGRB or soft gamma repeater (SGR) events, and reclassification of seven events (originally flagged as SGRB by the clustering algorithm) to LGRBs. The result is a reduction in the size of the original LGRB sample from 1376 to 1366 (Table 1). It is notable that the inclusion of the uncertainties on the two GRB variables $T_{90}$ and $E_{p}$ turns out to not have significant effects on the derived samples of the two GRB classes discussed above. Also, a classification based on $T_{50}$ instead of $T_{90}$ results in about the same samples for the two GRB classes with only a negligible difference of $\sim 0.7 \%$.

\subsection{Model Construction}

The goal of the presented analysis is to derive a multivariate model that is capable of reproducing the observational data of the 1366 BATSE LGRBs. Examples of multivariate treatment of LGRB data are rare in GRB literature, with the most recent (and perhaps the only) example of such work presented by B10. Conversely, many authors have focused primarily on the univariate distribution of the spectral parameters, most importantly on the LF. A variety of univariate models have been proposed as the LGRB LF and fit to data by approximating the complex detector threshold as a step function (Schmidt 1999) or an efficiency grid (e.g., the four-interval efficiency modeling of Guetta et al. 2005) or by other approximation methods. A more accurate modeling of the LF, however, requires at least two LGRB observables incorporated in the model: the bolometric peak flux $\left(P_{\text {bol }}\right)$ and the observed peak energy $\left(E_{p}\right)$. The parameter $E_{p}$ is required, since most gamma-ray detectors are photon counters, a quantity that depends on not only $P_{\text {bol }}$ but also $E_{p}$ of the burst. This leads to the requirement of using a bivariate distribution as the minimum acceptable model to begin with, for the purpose of constraining the LF. The choice of model can be almost anything (e.g., Kommers et al. 2000; Porciani \& Madau 2001; Sethi \& Bhargavi 2001; Schmidt 2009; Campisi et al. 2010; Wang \& Dai 2011), since current theories of LGRB prompt emission do not set strong limits on the shape and range of the LF or any other LGRB spectral or temporal variables.

\footnotetext{
1 Data for 1966 BATSE GRBs with firmly measured peak flux, fluence, and duration are taken from The BATSE Gamma Ray Burst Catalogs: http://www.batse.msfc.nasa.gov/batse/grb/catalog/. The spectral peak energy $\left(E_{p}\right)$ estimates of these events are taken from Shahmoradi \& Nemiroff (2010), also available for download at https://sites.google.com/site/amshportal/ research/aca/in-the-news/lgrb-world-model.
} 
Table 1

1366 BATSE Catalog Triggers Classified as LGRBs

\begin{tabular}{|c|c|c|c|c|c|c|c|c|c|c|c|c|c|}
\hline Trigger & Trigger & Trigger & Trigger & Trigger & Trigger & Trigger & Trigger & Trigger & Trigger & Trigger & Trigger & Trigger & Trigger \\
\hline 105 & 107 & 109 & 110 & 111 & 114 & 121 & 130 & 133 & 143 & 148 & 160 & 171 & 179 \\
\hline 204 & 211 & 214 & 219 & 222 & 223 & 226 & 228 & 235 & 237 & 249 & 257 & 288 & 332 \\
\hline 351 & 394 & 398 & 401 & 404 & 408 & 414 & 451 & 465 & 467 & 469 & 472 & 473 & 493 \\
\hline 501 & 516 & 526 & 540 & 543 & 548 & 549 & 559 & 563 & 577 & 591 & 594 & 606 & 630 \\
\hline 647 & 658 & 659 & 660 & 673 & 676 & 678 & 680 & 685 & 686 & 690 & 692 & 704 & 717 \\
\hline 741 & 752 & 753 & 755 & 761 & 764 & 773 & 795 & 803 & 815 & 816 & 820 & 824 & 825 \\
\hline 829 & 840 & 841 & 869 & 907 & 914 & 927 & 938 & 946 & 973 & 999 & 1009 & 1036 & 1039 \\
\hline 1042 & 1046 & 1085 & 1086 & 1087 & 1114 & 1120 & 1122 & 1123 & 1125 & 1126 & 1141 & 1145 & 1148 \\
\hline 1150 & 1152 & 1153 & 1156 & 1157 & 1159 & 1167 & 1190 & 1192 & 1196 & 1197 & 1200 & 1204 & 1213 \\
\hline 1218 & 1221 & 1235 & 1244 & 1279 & 1288 & 1291 & 1298 & 1303 & 1306 & 1318 & 1382 & 1384 & 1385 \\
\hline 1390 & 1396 & 1406 & 1416 & 1419 & 1425 & 1432 & 1439 & 1440 & 1446 & 1447 & 1449 & 1452 & 1456 \\
\hline 1458 & 1467 & 1468 & 1472 & 1492 & 1515 & 1533 & 1540 & 1541 & 1551 & 1552 & 1558 & 1559 & 1561 \\
\hline 1567 & 1574 & 1578 & 1579 & 1580 & 1586 & 1590 & 1601 & 1604 & 1606 & 1609 & 1611 & 1614 & 1623 \\
\hline 1625 & 1626 & 1628 & 1642 & 1646 & 1651 & 1652 & 1653 & 1655 & 1656 & 1657 & 1660 & 1661 & 1663 \\
\hline 1664 & 1667 & 1676 & 1687 & 1693 & 1700 & 1701 & 1704 & 1711 & 1712 & 1714 & 1717 & 1730 & 1731 \\
\hline 1733 & 1734 & 1740 & 1742 & 1806 & 1807 & 1815 & 1819 & 1830 & 1883 & 1885 & 1886 & 1922 & 1924 \\
\hline 1956 & 1967 & 1974 & 1982 & 1989 & 1993 & 1997 & 2018 & 2019 & 2035 & 2047 & 2053 & 2061 & 2067 \\
\hline 2069 & 2070 & 2074 & 2077 & 2079 & 2080 & 2081 & 2083 & 2087 & 2090 & 2093 & 2101 & 2102 & 2105 \\
\hline 2106 & 2110 & 2111 & 2112 & 2114 & 2119 & 2122 & 2123 & 2129 & 2133 & 2138 & 2140 & 2143 & 2148 \\
\hline 2149 & 2151 & 2152 & 2156 & 2181 & 2187 & 2188 & 2189 & 2190 & 2191 & 2193 & 2197 & 2202 & 2203 \\
\hline 2204 & 2207 & 2211 & 2213 & 2219 & 2228 & 2230 & 2232 & 2233 & 2240 & 2244 & 2252 & 2253 & 2254 \\
\hline 2267 & 2276 & 2277 & 2287 & 2298 & 2304 & 2306 & 2309 & 2310 & 2311 & 2315 & 2316 & 2321 & 2324 \\
\hline 2325 & 2328 & 2329 & 2340 & 2344 & 2345 & 2346 & 2347 & 2349 & 2362 & 2367 & 2371 & 2373 & 2375 \\
\hline 2380 & 2381 & 2383 & 2385 & 2387 & 2391 & 2392 & 2393 & 2394 & 2405 & 2419 & 2423 & 2428 & 2429 \\
\hline 2430 & 2432 & 2435 & 2436 & 2437 & 2438 & 2440 & 2441 & 2442 & 2443 & 2446 & 2447 & 2450 & 2451 \\
\hline 2452 & 2453 & 2458 & 2460 & 2472 & 2476 & 2477 & 2482 & 2484 & 2495 & 2496 & 2500 & 2505 & 2508 \\
\hline 2510 & 2511 & 2515 & 2519 & 2522 & 2528 & 2530 & 2533 & 2537 & 2541 & 2551 & 2560 & 2569 & 2570 \\
\hline 2581 & 2586 & 2589 & 2593 & 2600 & 2603 & 2606 & 2608 & 2610 & 2611 & 2619 & 2620 & 2628 & 2634 \\
\hline 2636 & 2640 & 2641 & 2660 & 2662 & 2663 & 2664 & 2665 & 2671 & 2677 & 2681 & 2688 & 2691 & 2695 \\
\hline 2696 & 2697 & 2700 & 2703 & 2706 & 2709 & 2711 & 2719 & 2725 & 2727 & 2736 & 2749 & 2750 & 2751 \\
\hline 2753 & 2767 & 2770 & 2774 & 2775 & 2780 & 2790 & 2793 & 2797 & 2798 & 2812 & 2815 & 2825 & 2830 \\
\hline 2831 & 2843 & 2848 & 2850 & 2852 & 2853 & 2855 & 2856 & 2857 & 2862 & 2863 & 2864 & 2877 & 2880 \\
\hline 2889 & 2890 & 2891 & 2897 & 2898 & 2900 & 2901 & 2913 & 2916 & 2917 & 2919 & 2922 & 2924 & 2925 \\
\hline 2927 & 2929 & 2931 & 2932 & 2944 & 2945 & 2947 & 2948 & 2950 & 2951 & 2953 & 2958 & 2961 & 2980 \\
\hline 2984 & 2985 & 2986 & 2990 & 2992 & 2993 & 2994 & 2996 & 2998 & 3001 & 3003 & 3005 & 3011 & 3012 \\
\hline 3015 & 3017 & 3026 & 3028 & 3029 & 3032 & 3035 & 3040 & 3042 & 3055 & 3056 & 3057 & 3067 & 3068 \\
\hline 3070 & 3071 & 3072 & 3074 & 3075 & 3076 & 3080 & 3084 & 3085 & 3088 & 3091 & 3093 & 3096 & 3100 \\
\hline 3101 & 3102 & 3103 & 3105 & 3109 & 3110 & 3115 & 3119 & 3120 & 3127 & 3128 & 3129 & 3130 & 3131 \\
\hline 3132 & 3134 & 3135 & 3136 & 3138 & 3139 & 3141 & 3142 & 3143 & 3153 & 3156 & 3159 & 3166 & 3167 \\
\hline 3168 & 3171 & 3174 & 3177 & 3178 & 3193 & 3212 & 3217 & 3220 & 3227 & 3229 & 3237 & 3238 & 3241 \\
\hline 3242 & 3245 & 3246 & 3247 & 3255 & 3256 & 3257 & 3259 & 3267 & 3269 & 3276 & 3279 & 3283 & 3284 \\
\hline 3287 & 3290 & 3292 & 3301 & 3306 & 3307 & 3319 & 3320 & 3321 & 3322 & 3324 & 3330 & 3336 & 3339 \\
\hline 3345 & 3347 & 3350 & 3351 & 3352 & 3356 & 3358 & 3364 & 3369 & 3370 & 3378 & 3403 & 3405 & 3407 \\
\hline 3408 & 3415 & 3416 & 3436 & 3439 & 3448 & 3458 & 3465 & 3471 & 3472 & 3480 & 3481 & 3485 & 3486 \\
\hline 3488 & 3489 & 3491 & 3493 & 3503 & 3505 & 3509 & 3511 & 3512 & 3514 & 3515 & 3516 & 3523 & 3527 \\
\hline 3528 & 3552 & 3567 & 3569 & 3588 & 3593 & 3598 & 3608 & 3618 & 3634 & 3637 & 3648 & 3649 & 3654 \\
\hline 3655 & 3658 & 3662 & 3663 & 3664 & 3671 & 3717 & 3733 & 3740 & 3745 & 3765 & 3766 & 3768 & 3771 \\
\hline 3773 & 3776 & 3779 & 3788 & 3792 & 3800 & 3801 & 3805 & 3807 & 3811 & 3814 & 3815 & 3819 & 3840 \\
\hline 3843 & 3853 & 3860 & 3869 & 3870 & 3871 & 3875 & 3879 & 3886 & 3890 & 3891 & 3892 & 3893 & 3899 \\
\hline 3900 & 3901 & 3903 & 3905 & 3906 & 3908 & 3909 & 3912 & 3913 & 3914 & 3916 & 3917 & 3918 & 3924 \\
\hline 3926 & 3929 & 3930 & 3935 & 3941 & 3954 & 4039 & 4048 & 4095 & 4146 & 4157 & 4216 & 4251 & 4312 \\
\hline 4350 & 4368 & 4388 & 4556 & 4569 & 4653 & 4701 & 4710 & 4745 & 4814 & 4939 & 4959 & 5080 & 5255 \\
\hline 5304 & 5305 & 5379 & 5387 & 5389 & 5407 & 5409 & 5411 & 5412 & 5415 & 5416 & 5417 & 5419 & 5420 \\
\hline 5421 & 5423 & 5428 & 5429 & 5433 & 5434 & 5447 & 5450 & 5451 & 5454 & 5463 & 5464 & 5465 & 5466 \\
\hline 5470 & 5472 & 5473 & 5474 & 5475 & 5476 & 5477 & 5478 & 5479 & 5480 & 5482 & 5483 & 5484 & 5486 \\
\hline 5487 & 5489 & 5490 & 5492 & 5493 & 5494 & 5495 & 5497 & 5503 & 5504 & 5507 & 5508 & 5510 & 5512 \\
\hline 5513 & 5515 & 5516 & 5517 & 5518 & 5523 & 5524 & 5526 & 5530 & 5531 & 5538 & 5539 & 5540 & 5541 \\
\hline 5542 & 5545 & 5548 & 5551 & 5554 & 5555 & 5559 & 5563 & 5565 & 5566 & 5567 & 5569 & 5571 & 5572 \\
\hline 5573 & 5574 & 5575 & 5581 & 5585 & 5589 & 5590 & 5591 & 5593 & 5594 & 5597 & 5601 & 5603 & 5604 \\
\hline 5605 & 5606 & 5608 & 5610 & 5612 & 5614 & 5615 & 5617 & 5618 & 5621 & 5622 & 5624 & 5626 & 5627 \\
\hline 5628 & 5632 & 5635 & 5637 & 5640 & 5644 & 5645 & 5646 & 5648 & 5654 & 5655 & 5667 & 5697 & 5704 \\
\hline 5706 & 5713 & 5715 & 5716 & 5718 & 5719 & 5721 & 5723 & 5725 & 5726 & 5729 & 5731 & 5736 & 5773 \\
\hline 5867 & 5890 & 5955 & 5983 & 5989 & 5995 & 6004 & 6082 & 6083 & 6090 & 6098 & 6100 & 6101 & 6102 \\
\hline 6103 & 6104 & 6111 & 6113 & 6115 & 6118 & 6119 & 6124 & 6127 & 6128 & 6131 & 6137 & 6139 & 6141 \\
\hline 6147 & 6151 & 6152 & 6154 & 6158 & 6159 & 6165 & 6167 & 6168 & 6176 & 6186 & 6188 & 6189 & 6190 \\
\hline 6194 & 6198 & 6206 & 6222 & 6223 & 6225 & 6226 & 6227 & 6228 & 6233 & 6234 & 6241 & 6242 & 6243 \\
\hline
\end{tabular}


Table 1

(Continued)

\begin{tabular}{|c|c|c|c|c|c|c|c|c|c|c|c|c|c|}
\hline Trigger & Trigger & Trigger & Trigger & Trigger & Trigger & Trigger & Trigger & Trigger & Trigger & Trigger & Trigger & Trigger & Trigger \\
\hline 6244 & 6249 & 6266 & 6267 & 6269 & 6270 & 6271 & 6272 & 6273 & 6274 & 6279 & 6280 & 6283 & 6285 \\
\hline 6288 & 6295 & 6298 & 6300 & 6303 & 6304 & 6305 & 6306 & 6308 & 6309 & 6315 & 6317 & 6319 & 6320 \\
\hline 6321 & 6322 & 6323 & 6328 & 6329 & 6330 & 6334 & 6335 & 6337 & 6339 & 6344 & 6345 & 6346 & 6349 \\
\hline 6351 & 6353 & 6355 & 6369 & 6370 & 6375 & 6380 & 6388 & 6390 & 6395 & 6396 & 6397 & 6399 & 6400 \\
\hline 6404 & 6405 & 6408 & 6409 & 6413 & 6414 & 6419 & 6422 & 6425 & 6435 & 6437 & 6440 & 6444 & 6446 \\
\hline 6448 & 6450 & 6451 & 6453 & 6454 & 6472 & 6487 & 6489 & 6490 & 6498 & 6504 & 6519 & 6520 & 6521 \\
\hline 6522 & 6523 & 6525 & 6528 & 6529 & 6531 & 6533 & 6534 & 6536 & 6538 & 6539 & 6544 & 6546 & 6550 \\
\hline 6551 & 6552 & 6554 & 6557 & 6560 & 6564 & 6566 & 6576 & 6577 & 6578 & 6582 & 6583 & 6585 & 6587 \\
\hline 6589 & 6590 & 6592 & 6593 & 6598 & 6600 & 6601 & 6602 & 6605 & 6610 & 6611 & 6613 & 6615 & 6616 \\
\hline 6619 & 6620 & 6621 & 6622 & 6625 & 6629 & 6630 & 6631 & 6632 & 6642 & 6648 & 6649 & 6655 & 6657 \\
\hline 6658 & 6665 & 6666 & 6670 & 6672 & 6673 & 6674 & 6676 & 6678 & 6683 & 6686 & 6694 & 6695 & 6698 \\
\hline 6702 & 6707 & 6708 & 6720 & 6745 & 6762 & 6763 & 6764 & 6767 & 6774 & 6782 & 6796 & 6802 & 6814 \\
\hline 6816 & 6830 & 6831 & 6853 & 6877 & 6880 & 6882 & 6884 & 6891 & 6892 & 6903 & 6911 & 6914 & 6917 \\
\hline 6930 & 6935 & 6938 & 6963 & 6987 & 6989 & 7000 & 7012 & 7028 & 7030 & 7064 & 7087 & 7108 & 7110 \\
\hline 7113 & 7116 & 7130 & 7147 & 7164 & 7167 & 7170 & 7172 & 7178 & 7183 & 7185 & 7191 & 7206 & 7207 \\
\hline 7209 & 7213 & 7219 & 7228 & 7230 & 7247 & 7250 & 7255 & 7263 & 7285 & 7293 & 7295 & 7298 & 7301 \\
\hline 7310 & 7318 & 7319 & 7322 & 7323 & 7328 & 7335 & 7343 & 7357 & 7358 & 7360 & 7369 & 7371 & 7374 \\
\hline 7376 & 7377 & 7379 & 7381 & 7386 & 7387 & 7390 & 7403 & 7404 & 7429 & 7432 & 7433 & 7446 & 7451 \\
\hline 7452 & 7457 & 7460 & 7464 & 7469 & 7475 & 7477 & 7481 & 7485 & 7486 & 7487 & 7488 & 7491 & 7493 \\
\hline 7494 & 7497 & 7500 & 7502 & 7503 & 7504 & 7509 & 7515 & 7517 & 7518 & 7520 & 7523 & 7527 & 7528 \\
\hline 7529 & 7532 & 7533 & 7535 & 7548 & 7549 & 7550 & 7551 & 7552 & 7560 & 7563 & 7564 & 7566 & 7567 \\
\hline 7568 & 7573 & 7575 & 7576 & 7579 & 7580 & 7587 & 7588 & 7597 & 7598 & 7603 & 7604 & 7605 & 7606 \\
\hline 7607 & 7608 & 7609 & 7614 & 7615 & 7617 & 7619 & 7625 & 7630 & 7635 & 7638 & 7642 & 7645 & 7648 \\
\hline 7654 & 7656 & 7657 & 7660 & 7662 & 7677 & 7678 & 7683 & 7684 & 7688 & 7695 & 7701 & 7703 & 7705 \\
\hline 7707 & 7711 & 7727 & 7729 & 7741 & 7744 & 7749 & 7750 & 7752 & 7762 & 7766 & 7769 & 7770 & 7780 \\
\hline 7781 & 7785 & 7786 & 7788 & 7790 & 7794 & 7795 & 7798 & 7802 & 7803 & 7810 & 7818 & 7822 & 7825 \\
\hline 7831 & 7835 & 7838 & 7840 & 7841 & 7843 & 7845 & 7858 & 7862 & 7868 & 7872 & 7884 & 7885 & 7886 \\
\hline 7888 & 7900 & 7902 & 7903 & 7906 & 7918 & 7923 & 7924 & 7929 & 7932 & 7934 & 7936 & 7938 & 7942 \\
\hline 7948 & 7954 & 7963 & 7968 & 7969 & 7973 & 7976 & 7984 & 7987 & 7989 & 7992 & 7994 & 7997 & 7998 \\
\hline 8001 & 8004 & 8008 & 8009 & 8012 & 8019 & 8022 & 8026 & 8030 & 8036 & 8039 & 8045 & 8049 & 8050 \\
\hline 8054 & 8059 & 8061 & 8062 & 8063 & 8064 & 8066 & 8073 & 8075 & 8084 & 8086 & 8087 & 8098 & 8099 \\
\hline 8101 & 8102 & 8105 & 8110 & 8111 & 8112 & 8116 & 8121 & - & - & - & - & - & - \\
\hline
\end{tabular}

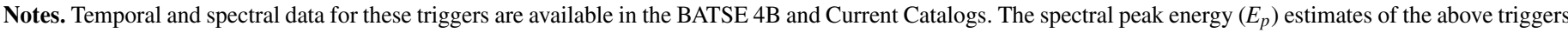

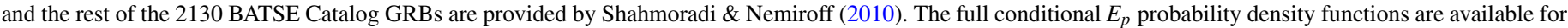
download at https://sites.google.com/site/amshportal/research/aca/in-the-news/lgrb-world-model.

Here, the multivariate log-normal distribution is proposed as the simplest natural candidate model capable of describing data. The motivation behind this choice of model comes from the available observational data that closely resemble a joint multivariate log-normal distribution for the four most widely studied temporal and spectral parameters of LGRBs in the observer frame: $P_{\mathrm{bol}}, S_{\mathrm{bol}}$ (bolometric fluence), $E_{p}, T_{90}$ : since most LGRBs originate from moderate redshifts $z \sim 1-3$, a fact known thanks to Swift satellite (e.g., B10; Racusin et al. 2011), the convolution of these observer-frame parameters with the redshift distribution results in negligible variation in the shape of the rest-frame joint distribution of the same LGRB parameters. Therefore, the redshift-convoluted four-dimensional (4D) restframe distribution can be well approximated as a linear translation from the observer-frame parameter space to the rest-frame parameter space, keeping the shape of the distribution almost intact. This implies that the joint distribution of the intrinsic LGRB variables: the isotropic peak luminosity $\left(L_{\text {iso }}\right)$, the total isotropic emission $\left(E_{\text {iso }}\right)$, the rest-frame time-integrated spectral peak energy $\left(E_{p, z}\right)$, and the rest-frame duration $\left(T_{90, z}\right)$ might be indeed well described by a multivariate log-normal distribution.

In general, models with higher nonzero moments than the log-normal model can also be considered for fitting, such as a multivariate skew-lognormal (e.g., Azzalini 1985) or variants of multivariate stable distributions (e.g., Press 1972). This, however, requires fitting for a higher number of free parameters, which is practically impossible given BATSE data with no available redshift information.

Following the discussion above, the process of LGRB observation can be therefore considered as a non-homogeneous Poisson process whose mean rate parameter-the cosmic LGRB differential rate, $\mathcal{R}_{\text {cosmic }}$-is the product of the differential comoving LGRB rate density $\dot{\zeta}(z)$ with a $p=4 \mathrm{D} \log$-normal probability density function (pdf), $\mathcal{L N}$, of four LGRB variables: $L_{\text {iso }}, E_{\text {iso }}, E_{p, z}$, and $T_{90, z}$, with location vector $\boldsymbol{\mu}$ and the scale (i.e., covariance) matrix $\Sigma$,

$$
\begin{aligned}
\mathcal{R}_{\text {cosmic }} & =\frac{d N}{d L_{\text {iso }} d E_{\text {iso }} d E_{p, z} d T_{90, z} d z} \\
& \propto \mathcal{L N}\left(L_{\text {iso }}, E_{\text {iso }}, E_{p, z}, T_{90, z} \mid \mu, \Sigma\right) \\
& \times \frac{\dot{\zeta}(z) d V / d z}{(1+z)},
\end{aligned}
$$

where the factor $(1+z)$ in the denominator accounts for cosmological time dilation and the comoving volume element per unit redshift, $d V / d z$, is

$$
\frac{d V}{d z}=\frac{C}{H_{0}} \frac{4 \pi D_{L}^{2}(z)}{(1+z)^{2}\left[\Omega_{M}(1+z)^{3}+\Omega_{\Lambda}\right]^{1 / 2}},
$$


with $D_{L}$ being the luminosity distance,

$$
D_{L}(z)=\frac{C}{H_{0}}(1+z) \int_{0}^{z} d z^{\prime}\left[\left(1+z^{\prime}\right)^{3} \Omega_{M}+\Omega_{\Lambda}\right]^{-1 / 2},
$$

assuming a flat $\Lambda \mathrm{CDM}$ cosmology, with parameters set to $h=0.70, \Omega_{\mathrm{M}}=0.27$, and $\Omega_{\Lambda}=0.73$ (Jarosik et al. 2011). Here, $C$ and $H_{0}=100 h\left(\mathrm{Km} \mathrm{s}^{-1} \mathrm{MPc}^{-1}\right)$ stand for the speed of light and the Hubble constant, respectively.

The 4D log-normal distribution of Equation (1), $\mathcal{L N}$, has an intimate connection to multivariate Gaussian distribution in the logarithmic space of LGRB observable parameters (cf. Appendix D).

One could generalize the LGRB rate of Equation (1) to incorporate a redshift evolution of the LGRB variables in the form of $\mu_{i}(z)=\mu_{0, i}+\alpha_{i} \log (1+z),(i=1, \ldots, 4)$, where $\boldsymbol{\alpha}$ has to be constrained by observational data. This is, however, impractical for BATSE data due to unknown redshifts, as the fitting results in degenerate values for $\boldsymbol{\alpha}$. Nevertheless, the multivariate analysis of Swift LGRBs presented by B10 strongly rejects the possibility of redshift evolution of the LF, a fact that further legitimizes the absence of redshift-luminosity evolution in Equation (1).

As for the comoving rate density $\dot{\zeta}(z)$, it is assumed that LGRBs trace the star formation rate (SFR) in the form of a piecewise power-law function of Hopkins \& Beacom (2006, hereafter HB06):

$$
\dot{\zeta}(z)=\frac{d N}{d z} \propto \begin{cases}(1+z)^{\gamma_{0}} & z<z_{0} \\ (1+z)^{\gamma_{1}} & z_{0}<z<z_{1} \\ (1+z)^{\gamma_{2}} & z>z_{1}\end{cases}
$$

with parameters $\left(z_{0}, z_{1}, \gamma_{0}, \gamma_{1}, \gamma_{2}\right)$ set to best-fit values $(0.97,4.5,3.4,-0.3,-7.8)$ of HB06, and also to the best values $(0.993,3.8,3.3,0.055,-4.46)$ of an updated SFR fit by $\mathrm{Li}$ (2008). Alternatively, the bias-corrected redshift distribution of LGRBs derived from Swift data (B10) with best-fit parameter values $(0.97,4.00,3.14,1.36,-2.92)$ can be employed as $\dot{\zeta}(z)$. This parameter set is consistent with an LGRB rate scenario tracing metallicity-corrected SFR with a cutoff $Z / Z_{\odot} \sim 0.2-0.5$ (Figure 10 and Equation (8) in B10; Li 2008). The hypothesis of LGRB rate evolving with cosmic metallicity is both predicted by the Collapsar model of LGRBs (e.g., Woosley \& Heger 2006) and supported by observations of LGRB host galaxies (e.g., Stanek et al. 2006; Levesque et al. 2010a), although the metallicity-rate connection and the presence of a sharp metallicity cutoff have been challenged by few recent host galaxy observations (e.g., Levesque et al. 2010c, 2010b) and possible unknown observational biases (e.g., Levesque 2012).

The cosmic LGRB rate, $\mathcal{R}_{\text {cosmic }}$, in Equation (1), although quantified correctly, does not represent the observed rate $\left(\mathcal{R}_{\text {obs }}\right)$ of LGRBs detected by BATSE LADs, unless convolved with an accurate model of BATSE trigger efficiency, $\eta$, as a function of the burst redshift and rest-frame parameters, discussed in Appendix B,

$$
\mathcal{R}_{\mathrm{obs}}=\eta\left(L_{\mathrm{iso}}, E_{p, z}, T_{90, z}, z\right) \times \mathcal{R}_{\text {cosmic }}
$$

\subsection{Model Fitting}

Now, with a statistical model for the observed rate of LGRBs at hand (i.e., Equation (5)), the best-fit parameters can be obtained by the method of maximum likelihood. This is done by maximizing the likelihood function of the model, given the
Table 2

\begin{tabular}{|c|c|c|c|}
\hline Parameter & HB06 & Li (2008) & B10 \\
\hline \multicolumn{4}{|c|}{ Redshift parameters (Equation (4)) } \\
\hline$z_{0}$ & 0.97 & 0.993 & 0.97 \\
\hline$z_{1}$ & 4.5 & 3.8 & 4.00 \\
\hline$\gamma_{0}$ & 3.4 & 3.3 & 3.14 \\
\hline$\gamma_{1}$ & -0.3 & 0.0549 & 1.36 \\
\hline$\gamma_{2}$ & -7.8 & -4.46 & -2.92 \\
\hline \multicolumn{4}{|c|}{ Location parameters } \\
\hline $\log \left(L_{\text {iso }}\right)$ & $51.35 \pm 0.20$ & $51.50 \pm 0.19$ & $51.73 \pm 0.19$ \\
\hline $\log \left(E_{\text {iso }}\right)$ & $51.82 \pm 0.20$ & $51.94 \pm 0.20$ & $52.03 \pm 0.21$ \\
\hline $\log \left(E_{p, z}\right)$ & $2.43 \pm 0.05$ & $2.47 \pm 0.05$ & $2.54 \pm 0.05$ \\
\hline $\log \left(T_{90, z}\right)$ & $0.99 \pm 0.03$ & $0.96 \pm 0.03$ & $0.80 \pm 0.03$ \\
\hline \multicolumn{4}{|c|}{ Scale parameters } \\
\hline $\log \left(\sigma_{L_{\text {iso }}}\right)$ & $-0.22 \pm 0.06$ & $-0.23 \pm 0.06$ & $-0.20 \pm 0.05$ \\
\hline $\log \left(\sigma_{E_{\text {iso }}}\right)$ & $-0.07 \pm 0.03$ & $-0.07 \pm 0.03$ & $-0.04 \pm 0.03$ \\
\hline $\log \left(\sigma_{E_{p, z}}\right)$ & $-0.44 \pm 0.02$ & $-0.44 \pm 0.02$ & $-0.44 \pm 0.02$ \\
\hline$\underline{\log \left(\sigma_{T_{90, z}}\right)}$ & $-0.38 \pm 0.01$ & $-0.39 \pm 0.01$ & $-0.40 \pm 0.01$ \\
\hline \multicolumn{4}{|c|}{ Correlation coefficients } \\
\hline$\rho_{L_{\text {iso }}-E_{\text {iso }}}$ & $0.93 \pm 0.01$ & $0.94 \pm 0.01$ & $0.96 \pm 0.01$ \\
\hline$\rho_{L_{\mathrm{iso}}-E_{p, z}}$ & $0.47 \pm 0.07$ & $0.45 \pm 0.07$ & $0.44 \pm 0.08$ \\
\hline$\rho_{L_{\text {iso }}-T_{90, z}}$ & $0.52 \pm 0.08$ & $0.59 \pm 0.09$ & $0.75 \pm 0.07$ \\
\hline$\rho_{E_{\text {iso }}-E_{p, z}}$ & $0.58 \pm 0.04$ & $0.58 \pm 0.04$ & $0.59 \pm 0.04$ \\
\hline$\rho_{E_{\text {iso }}-T_{90, z}}$ & $0.63 \pm 0.05$ & $0.66 \pm 0.05$ & $0.74 \pm 0.04$ \\
\hline$\underline{\rho_{E_{p, z}}-T_{90, z}}$ & $0.34 \pm 0.04$ & $0.37 \pm 0.04$ & $0.50 \pm 0.04$ \\
\hline \multicolumn{4}{|c|}{ BATSE LGRB detection efficiency (Equation (A5)) } \\
\hline$\mu_{\text {thresh }}$ & $-0.44 \pm 0.02$ & $-0.45 \pm 0.02$ & $-0.44 \pm 0.02$ \\
\hline $\log \left(\sigma_{\text {thresh }}\right)$ & $-0.88 \pm 0.05$ & $-0.90 \pm 0.05$ & $-0.88 \pm 0.05$ \\
\hline
\end{tabular}

Mean Best-fit Parameters of LGRB World Model, for the Three Redshift Distribution Scenarios

Notes. The full Markov chain sampling of the above parameters from the 16-dimensional parameter space of the likelihood function (Appendix C) is available for download at https://sites.google.com/site/amshportal/research/ aca/in-the-news/lgrb-world-model for each of the three redshift distributions.

observational data, using a variant of the Metropolis-Hastings Markov chain Monte Carlo (MCMC) algorithm discussed in detail in Appendix C. As mentioned before, the fitting is performed for three redshift-distribution scenarios of HB06, B10, and Li (2008).

It is also known that the $T_{90}$ of LGRBs are potentially subject to estimation biases. To ensure that the reported $T_{90}$ of BATSE LGRBs do not bias the fitting results for the rest of the parameters, model fitting was also performed by considering only three spectral variables of BATSE LGRBs: the bolometric $1 \mathrm{~s}$ peak flux $\left(P_{\text {bol }}\right)$, bolometric fluence $\left(S_{\text {bol }}\right)$, and observed peak energy $\left(E_{p}\right)$, excluding duration $\left(T_{90, z}\right)$ variable from the model, thus reducing the dimension of the model by one. Only after the fitting was performed did it become clear that the inclusion of the $T_{90}$ of BATSE LGRBs in the fitting does not significantly affect the resulting best-fit parameters of the model. Therefore, only results from the full model fitting are presented here, as in Table 2.

Due to lack of redshift information for BATSE GRBs, the resulting parameters of the model exhibit strong covariations with each other. This is illustrated in the example correlation matrix of the LGRB world model in Table 3. All location parameters appear to correlate strongly positively with each other, and so do the scale parameters. The location parameters however negatively correlate with the scale parameters, meaning 
Correlation Matrix of the Parameters of the LGRB World Model, for the Median Case of an LGRB Cosmic Rate Tracing Star Formation Rate in Li (2008)

\begin{tabular}{|c|c|c|c|c|c|c|c|c|c|c|c|c|c|c|c|c|}
\hline Parameter & $\log \left(L_{\text {iso }}\right)$ & $\log \left(E_{\text {iso }}\right)$ & $\log \left(E_{p, z}\right)$ & $\log \left(T_{90, z}\right)$ & $\log \left(\sigma_{L_{\text {iso }}}\right)$ & $\log \left(\sigma_{E_{\text {iso }}}\right)$ & $\log \left(\sigma_{E_{p, z}}\right)$ & $\log \left(\sigma_{T_{90, z}}\right)$ & $\rho_{L_{\text {iso }}-E_{\text {iso }}}$ & $\rho_{L_{\text {iso }}-E_{p, z}}$ & $\rho_{L_{\text {iso }}-T_{90, z}}$ & $\rho_{E_{\text {iso }}-E_{p, z}}$ & $\rho_{E_{\text {iso }}-T_{90, z}}$ & $\rho_{E_{p, z}-T_{90, z}}$ & $\mu_{\text {thresh }}$ & $\log \left(\sigma_{\text {thresh }}\right)$ \\
\hline $\log \left(L_{\text {iso }}\right)$ & 1.00 & 0.99 & 0.90 & 0.34 & -0.91 & -0.86 & -0.59 & -0.14 & -0.10 & -0.45 & 0.51 & -0.52 & 0.45 & 0.05 & -0.68 & -0.44 \\
\hline $\log \left(E_{\text {iso }}\right)$ & & 1.00 & 0.92 & 0.42 & -0.91 & -0.90 & -0.62 & -0.15 & -0.20 & -0.54 & 0.45 & -0.56 & 0.40 & 0.00 & -0.67 & -0.43 \\
\hline $\log \left(E_{p, z}\right)$ & & & 1.00 & 0.38 & -0.82 & -0.84 & -0.79 & -0.15 & -0.26 & -0.77 & 0.40 & -0.77 & 0.38 & 0.07 & -0.61 & -0.40 \\
\hline $\log \left(T_{90, z}\right)$ & & & & 1.00 & -0.37 & -0.52 & -0.32 & -0.16 & -0.52 & -0.32 & -0.48 & -0.33 & -0.50 & -0.56 & -0.17 & -0.09 \\
\hline $\log \left(\sigma_{L_{\text {iso }}}\right)$ & & & & & 1.00 & 0.94 & 0.59 & 0.12 & 0.14 & 0.53 & -0.53 & 0.57 & -0.46 & 0.03 & 0.50 & 0.30 \\
\hline$\underline{\log \left(\sigma_{E_{\text {iso }}}\right)}$ & & & & & & 1.00 & 0.67 & 0.17 & 0.41 & 0.63 & -0.33 & 0.66 & -0.32 & 0.14 & 0.46 & 0.28 \\
\hline$\underline{\log \left(\sigma_{E_{p, z}}\right)}$ & & & & & & & 1.00 & 0.12 & 0.36 & 0.84 & -0.22 & 0.84 & -0.25 & -0.06 & 0.37 & 0.24 \\
\hline $\log \left(\sigma_{T_{90, z}}\right)$ & & & & & & & & 1.00 & -0.03 & 0.10 & -0.09 & 0.11 & 0.00 & 0.03 & 0.11 & 0.07 \\
\hline$\rho_{L_{\text {iso }}-E_{\text {iso }}}$ & & & & & & & & & 1.00 & 0.47 & 0.43 & 0.41 & 0.22 & 0.26 & -0.01 & -0.01 \\
\hline$\rho_{L_{\mathrm{iso}}-E_{p, z}}$ & & & & & & & & & & 1.00 & -0.15 & 0.97 & -0.21 & -0.05 & 0.29 & 0.19 \\
\hline$\rho_{L_{\text {iso }}-T_{90, z}}$ & & & & & & & & & & & 1.00 & -0.18 & 0.95 & 0.57 & -0.32 & -0.20 \\
\hline$\underline{\rho_{E_{\text {iso }}}-E_{p, z}}$ & & & & & & & & & & & & 1.00 & -0.22 & 0.06 & 0.29 & 0.18 \\
\hline$\underline{\rho_{E_{\text {iso }}-T_{90, z}}}$ & & & & & & & & & & & & & 1.00 & 0.64 & -0.26 & -0.17 \\
\hline$\underline{\rho_{E_{p, z}}-T_{90, z}}$ & & & & & & & & & & & & & & 1.00 & -0.09 & -0.07 \\
\hline$\mu_{\text {thresh }}$ & & & & & & & & & & & & & & & 1.00 & 0.79 \\
\hline $\log \left(\sigma_{\text {thresh }}\right)$ & & & & & & & & & & & & & & & & 1.00 \\
\hline
\end{tabular}


that an increase in the average values of the rest-frame parameters reduces the half-width of the corresponding distributions of the variables. In general, it is also observed that the correlations among the four variables weaken with increasing the location parameters. An exception to this is the correlation of $T_{90, z}$ with $L_{\text {iso }}$ and $E_{\text {iso }}$ which tends to increase with location parameters. Since an excess in the cosmic rates of LGRBs at high redshifts generally results in an increase in the values of location parameters, it can be said that "given BATSE LGRBs data, a higher rate for LGRBs at distant universe generally implies weaker $E_{\mathrm{iso}}-E_{p, z}$ and $L_{\mathrm{iso}}-E_{p, z}$ correlations and stronger covariation of $T_{90, z}$ with the three other parameters."

\subsection{Goodness-of-fit Tests}

In any statistical fitting problem, perhaps more important than the model construction is to provide tests showing how good the model fit is to input data. For many univariate studies of the GRB LF, this is done by employing well-established statistical tests such as the Kolmogorov-Smirnov (K-S; e.g., Kolmogoroff 1941; Smirnov 1948) or Pearson's $\chi^{2}$ (e.g., Fisher 1924) tests. In the case of multivariate studies (e.g., B10), a combination of visual inspection of the fitting results, K-S test on the marginal, and bivariate distributions and variants of $\chi^{2}$ (e.g., likelihood ratio) tests have been used.

In general, univariate tests on the marginal distributions of multivariate fits provide only necessary-but not sufficientevidence for a good multivariate fit. Alternatively one could assess the similarity by using nonparametric multivariate $\mathrm{GoF}$ tests. Such tests, although existing, have been rarely discussed and treated in statistics due to difficulties in the interpretation of the test statistic (e.g., Peacock 1983; Press et al. 1992; Justel et al. 1997). Ideally, one can always use the Pearson's $\chi^{2}$ GoF test for any multivariate distribution. However, for the special case of BATSE 1366 LGRBs, one would need an observed sample consisting of $N \gg 1366$ observations to avoid serious instabilities that occur in $\chi^{2}$ tests due to small sample sizes (e.g., Cochran 1954).

To ensure a good fit to the observational data in all-and not only univariate-levels of the multivariate structure of data, an assessment of similarity can be obtained by scanning and comparing the model and data along their principal axes, in addition to univariate tests on the marginal distributions. Although statistically not a sufficient condition for the multivariate similarity of the model prediction to data, this can provide strong evidence in favor of a good fit, at a much higher confidence than tests performed only on the marginal distributions.

Following from above, Figure 2 presents the model predictions for marginal distributions of the four LGRB variables in the observer frame. The K-S test probabilities for the similarity of the model predictions to the marginal distributions of BATSE LGRB variables are also reported on the top right of each plot. All three redshift-distribution parameters of HB06, Li (2008), and B10 (Equation (4)) result in relatively similar fits to BATSE data in the observer frame. Thus, for brevity, only plots for one representative (median) redshift distribution (i.e., Li 2008) are presented.

As the second level of GoF tests, the joint bivariate model predictions are compared to BATSE LGRB data, presented in Figures 3-5. This method of scanning model and data along the principal axes of the joint bivariate distributions can be generalized to trivariate and quadruvariate joint distributions. For brevity, however, only the bivariate tests are presented.

\section{RESULTS AND DISCUSSION}

It is observed in the plots of Figures 2-5 that the model provides excellent fit to data, within the uncertainties caused by random Poisson fluctuations in the BATSE LGRB observed rate. These random fluctuations in BATSE detections are encompassed in each graph by the green dashed lines that represent the $90 \%$ confidence intervals (CI) on BATSE LGRB detections (blue solid lines), derived by repeated sampling from the model.

Unfortunately, the same methods for a comparison of data and model cannot be applied in the LGRB rest frame, due to lack of redshift information for the BATSE sample of LGRBs. Nevertheless, a comparison of the model with observational data of other instruments - with measured redshifts - can provide clues on the underlying joint distribution of LGRB temporal and spectral variables in the rest frame compared to LGRB detections of different gamma-ray instruments, as will be done in the following sections.

\subsection{LGRB Luminosity Function and $\log (N)-\log (P)$ Diagram}

The $\log (N)-\log (P)$ diagram of GRBs has been subject of numerous studies in the BATSE era, primarily for the purpose of finding signatures of cosmological (versus galactic) origins in the LGRB rate. The cosmological origin of LGRBs is now well established. Nevertheless, the $\log (N)-\log (P)$ diagram can still provide useful information for future gamma-ray experiments.

Figure 2 (bottom) depicts the prediction of the LGRB world model for the traditional $\log (N)-\log (P)$ diagram for $1 \mathrm{~s}$ peak photon flux in the BATSE nominal detection energy range $50-300 \mathrm{keV}$, both for the differential (left panel) and the cumulative (right panel) LGRB rate. For all three LGRB cosmic rates considered in this work-as in Table 2-the differential $\log (N)-\log (P)$ diagram shows a peak in the rate at $P_{50-300} \sim 0.1$ photons s${ }^{-1} \mathrm{~cm}^{-2}$. Such a peak in the LGRB rate results in a relative flattening at the dim end of the cumulative $\log (N)-\log (P)$ diagram, as compared to its bright end. This observation has already been reported by B10 for the Swift sample of LGRBs, although an entirely different LF- a broken power-law LF-was used by B10 in their multivariate LGRB world model (cf. Equation (2) in B10).

On the other hand, the peak in the observer-frame LGRB rate translates to three relatively different (at $\sim 1 \sigma$ level) peaks in the LF of LGRBs. In general, it is observed that the peak of the LF (i.e., the average 1 s peak luminosity of LGRBs) increases with increasing cosmic rate of LGRBs at high redshift. This effect is well depicted in the top left panel of Figure 6 for the three LGRB cosmic rates considered: HB06, Li (2008), and B10.

Compared to the predictions of B10's LGRB world model (cf. bottom plot of Figure 6 in B10), the log-normal model suggests a lower peak for BATSE LGRB LF ( $\sim 52.3$ here versus $\sim 52.7$ in B10) for the same redshift distribution of LGRBs. Averaging over the three redshift distributions considered, the model predicts a dynamic $3 \sigma$ range of observer-frame brightness $\log \left(P_{\mathrm{bol}}\left(\mathrm{erg} \mathrm{s}^{-1} \mathrm{~cm}^{-2}\right)\right) \in[-7.11 \pm 2.66]$ corresponding to $P_{\text {bol }}\left(\mathrm{erg} \mathrm{s}^{-1} \mathrm{~cm}^{-2}\right) \in\left[1.70 \times 10^{-10}, 3.58 \times 10^{-5}\right]$ for LGRBs. This translates to an average dynamic $3 \sigma$ range-in the rest frame-of $\log \left(L_{\text {iso }}\left(\mathrm{erg} \mathrm{s}^{-1}\right)\right) \in[51.53 \pm 1.99]$ corresponding to $L_{\text {iso }}\left(\mathrm{erg} \mathrm{s}^{-1}\right) \in\left[3.46 \times 10^{49}, 3.38 \times 10^{53}\right]$.

\subsection{Isotropic Emission and Peak Energy Distributions}

A comparison of the top-right panel of Figure 6 with Swift observations of LGRBs (e.g., B10, Figures 7 and 8) indicates 

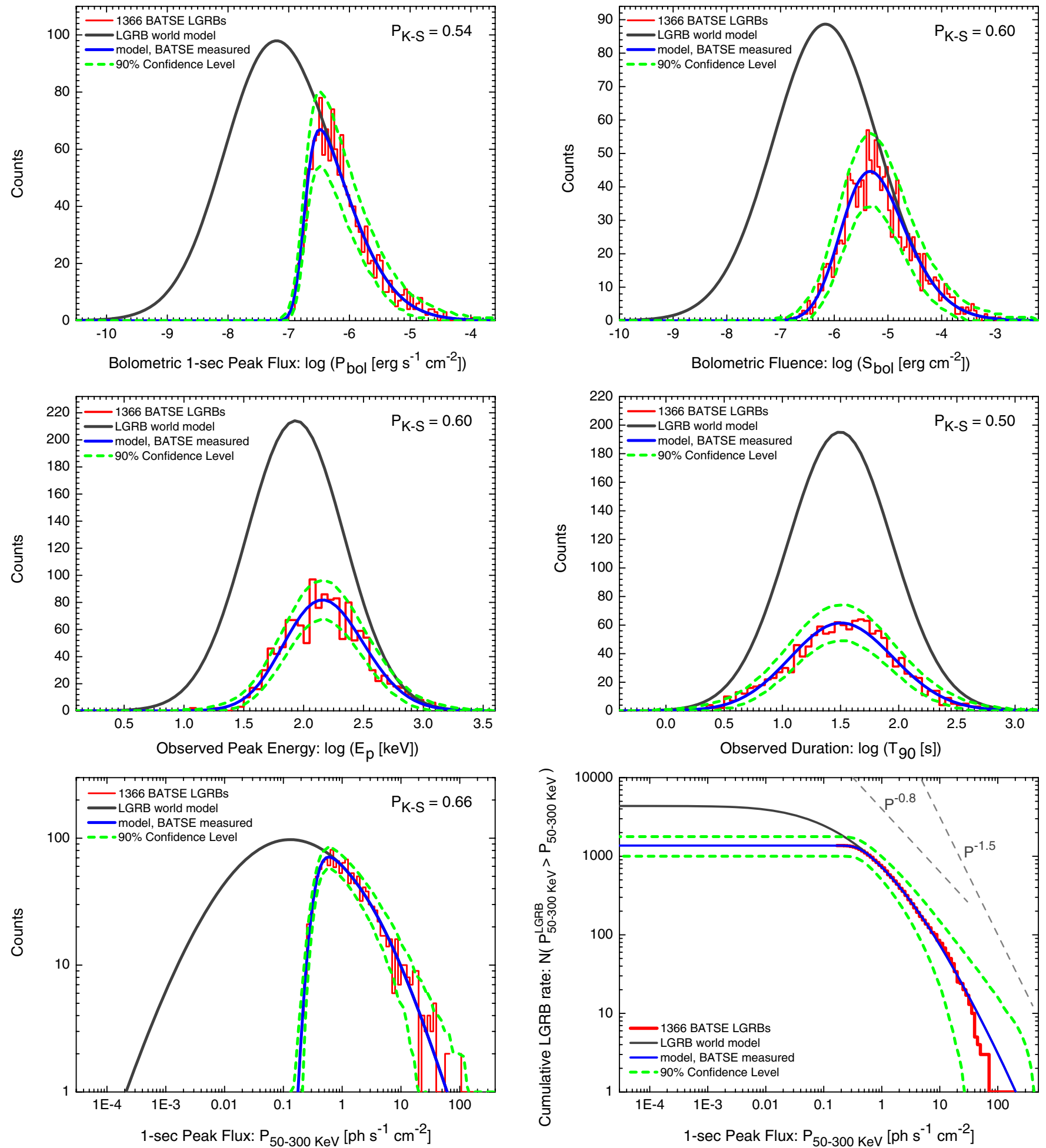

Figure 2. Marginal distribution predictions (solid blue lines) of the LGRB world model given BATSE detection efficiency, superposed on BATSE 1366 LGRB data (red histograms). The solid gray lines represent the model predictions for the entire LGRB population (detected and undetected), with no correction for BATSE sky exposure and the beaming factor $\left(f_{b}\right)$. The $90 \%$ confidence intervals (dashed green lines) represent random Poisson fluctuations expected in the BATSE LGRB-detection process. Bottom: the differential (left panel) and cumulative (right panel) rate of LGRBs as a function of peak photon flux in the BATSE nominal detection energy range (50-300 keV). For a comparison with the Swift sample of LGRBs and the proposed multivariate model of B10, the two dashed gray lines-taken from Figure 1 in B10-represent the predictions of the broken power-law luminosity function in Equation (2) of B10, in the observer frame. The Kolmogorov-Smirnov (K-S) test probabilities for the goodness-of-fit of the model predictions to BATSE data are reported at the top-right corner of each plot.

(A color version of this figure is available in the online journal.)

the similarity of the peak rates in BATSE and Swift samples of total isotropic emission $\left(E_{\text {iso }}\right)$ distributions. The distributions for both instruments show a similar peak at $\log \left(E_{\text {iso }}\right) \sim$ 52.7. The Swift $E_{\text {iso }}$ distribution, however, spans relatively lower $E_{\text {iso }}$ as compared to BATSE. This observation is not surprising if one takes into account the strong trivariate correlations of the three LGRB variables: $L_{\text {iso }}, E_{\text {iso }}$, and $E_{p, z}$, all of which play a role in LGRB detection by Swift 

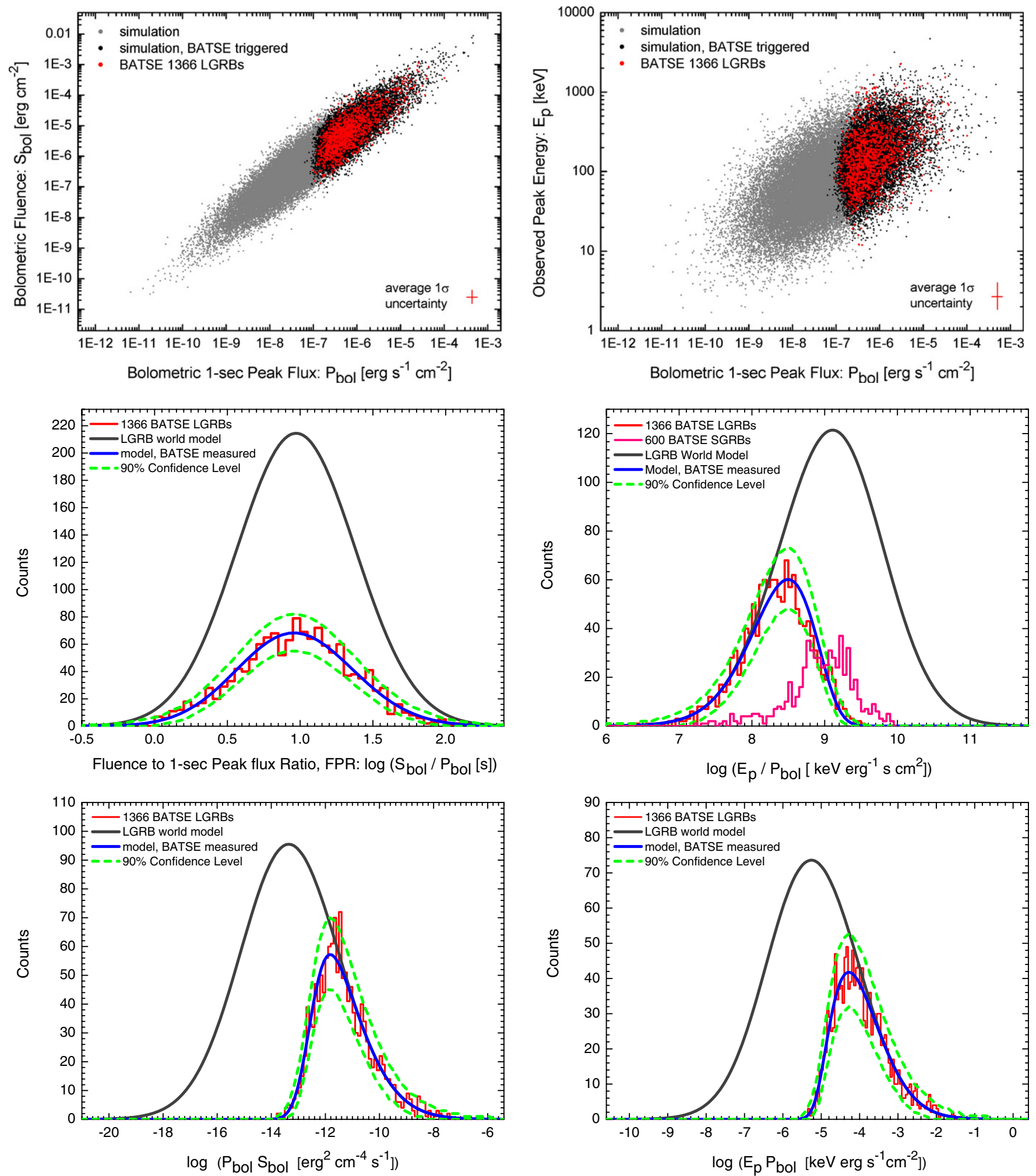

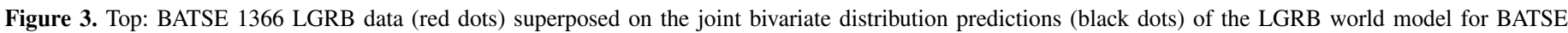

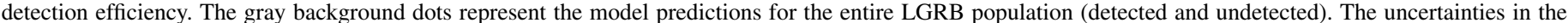

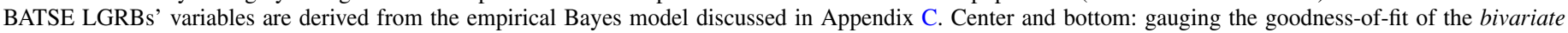

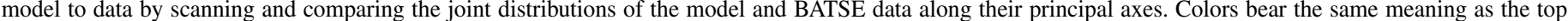
panels, in addition to the $90 \%$ confidence intervals (green dashed lines) that represent random Poisson fluctuations expected in BATSE LGRB-detection process.

(A color version of this figure is available in the online journal.)

and BATSE. In fact, a comparison of the spectral peak energy $\left(E_{p, z}\right)$ distributions of Swift (e.g., Figure 2 in B10) with the model's prediction for BATSE LGRBs (center left panel of Figure 6) reveals the relatively high sensitivity of the Swift BAT detector to dim soft LGRBs as compared to BATSE LADs. Such difference between the two detectors has already been discussed frequently by different authors (e.g., Band 2003, 2006). 

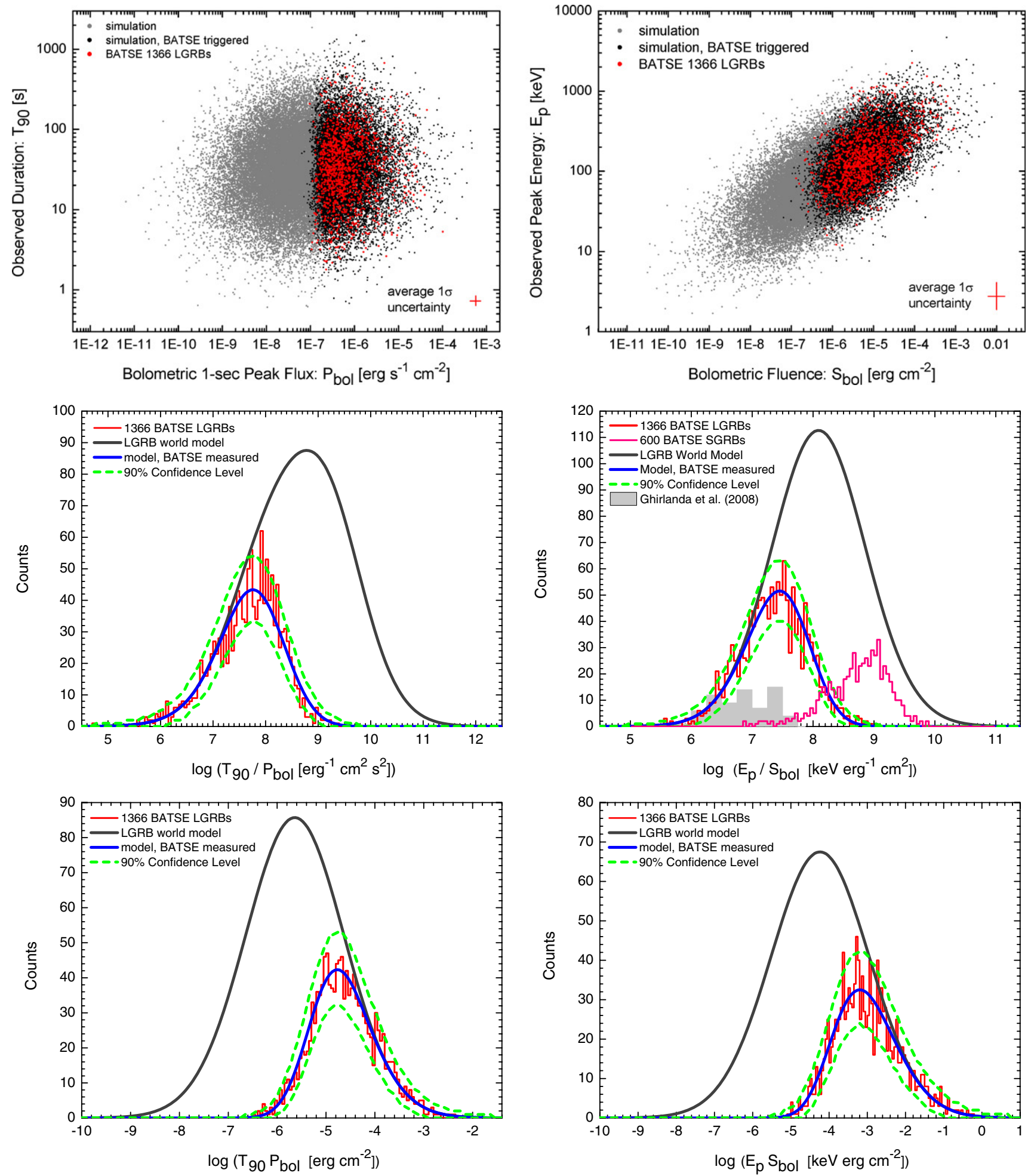

Figure 4. Top: BATSE 1366 LGRB data (red dots) superposed on the joint bivariate distribution predictions (black dots) of the LGRB world model for BATSE detection efficiency. The background gray dots represent the model predictions for the entire LGRB population (detected and undetected). The uncertainties in the BATSE LGRBs' variables are derived from the empirical Bayes model discussed in Appendix C. Center and bottom: gauging the goodness-of-fit of the bivariate model to data by scanning and comparing the joint distributions of the model and BATSE data along their principal axes. Colors have the same meaning as the top panels, in addition to the $90 \%$ confidence intervals (green dashed lines) that represent random Poisson fluctuations expected in the BATSE LGRB-detection process.

(A color version of this figure is available in the online journal.)

In sum, averaging over the three redshift distributions considered, the LGRB world model predicts a dynamic $3 \sigma$ range of observer-frame bolometric LGRB fluence $\log \left(S_{\text {bol }}\left(\mathrm{erg} \mathrm{cm}^{-2}\right)\right) \in[-6.16 \pm 3.01]$ corresponding to $S_{\text {bol }}\left(\mathrm{erg} \mathrm{cm}^{-2}\right) \in\left[6.82 \times 10^{-10}, 7.01 \times 10^{-4}\right]$. This translates to an average $3 \sigma$ range -in the rest frame - of $\log \left(E_{\text {iso }}(\mathrm{erg})\right) \in$ $[51.93 \pm 2.71]$ corresponding to $E_{\text {iso }}(\mathrm{erg}) \in\left[1.66 \times 10^{49}\right.$, $4.46 \times 10^{54}$.

As for the spectral peak energy ( $E_{p}$ and $\left.E_{p, z}\right)$ distributions, the model predicts a $3 \sigma$ range of observer-frame LGRB spectral 

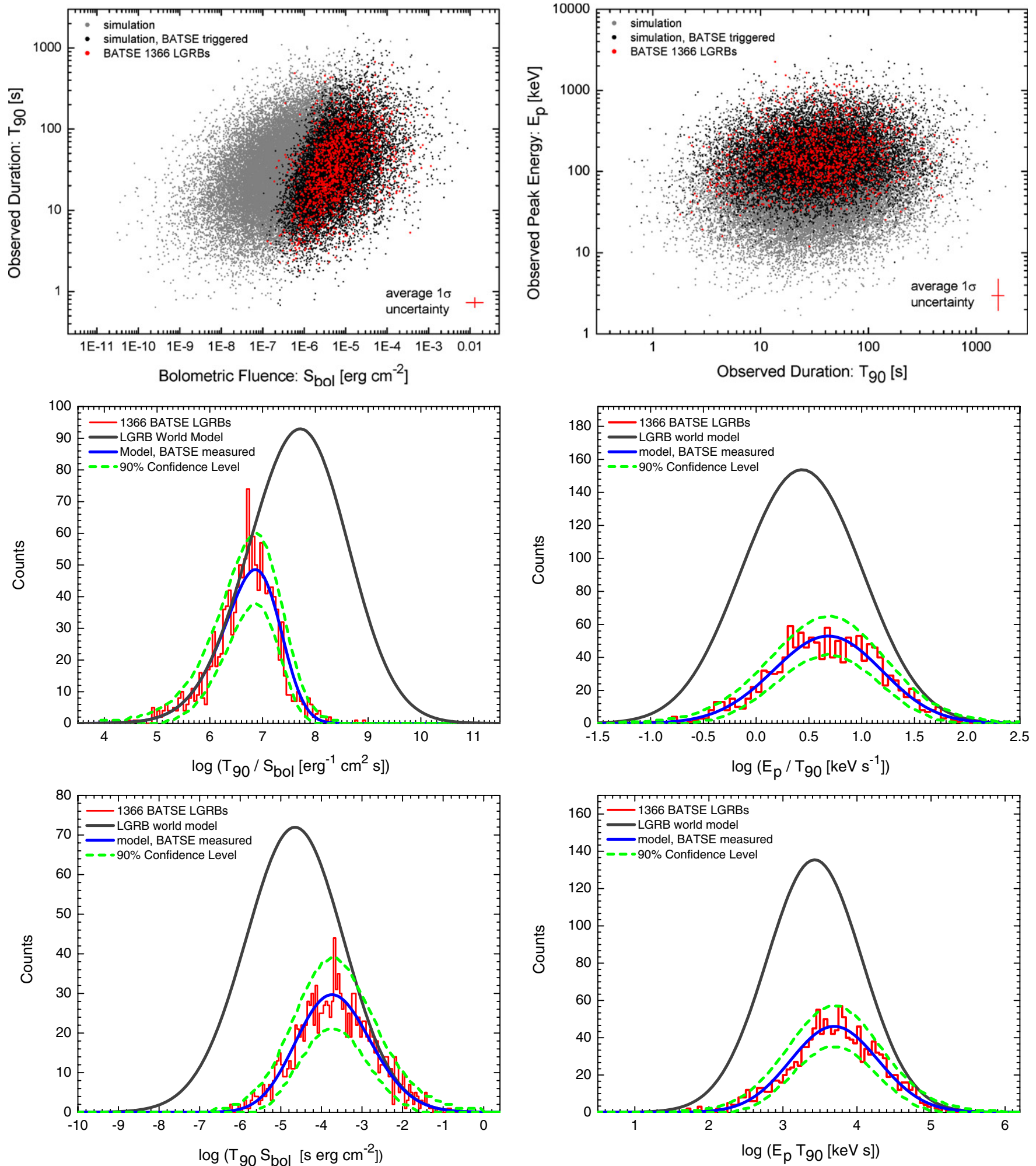

Figure 5. Top: BATSE 1366 LGRB data (red dots) superposed on the joint bivariate distribution predictions (black dots) of the LGRB world model for BATSE detection efficiency. The gray background dots represent the model predictions for the entire LGRB population (detected and undetected). The uncertainties in BATSE LGRBs' variables are derived from the empirical Bayes model discussed in Appendix C. Center and bottom: gauging the goodness-of-fit of the bivariate model to data by scanning and comparing the joint distributions of the model and BATSE data along their principal axes. Colors have the same meaning as the top panels, in addition to the $90 \%$ confidence intervals (green dashed lines) that represent random Poisson fluctuations expected in the BATSE LGRB-detection process.

(A color version of this figure is available in the online journal.)

peak energy $\log \left(E_{p}(\mathrm{keV})\right) \in[1.93 \pm 1.22]$ corresponding to $E_{p}(\mathrm{keV}) \in[5,1427]$. This translates to an average $3 \sigma$ range-in the rest frame- of $\log \left(E_{p, z}(\mathrm{keV})\right) \in[2.48 \pm 1.12]$ corresponding to $E_{p, z}(\mathrm{keV}) \in[23,4006]$.

\subsection{Duration Distribution}

GRBs are traditionally flagged as a long-duration class of bursts if their observed durations $\left(T_{90}\right)$ exceed $2 \mathrm{~s}$ (e.g., 

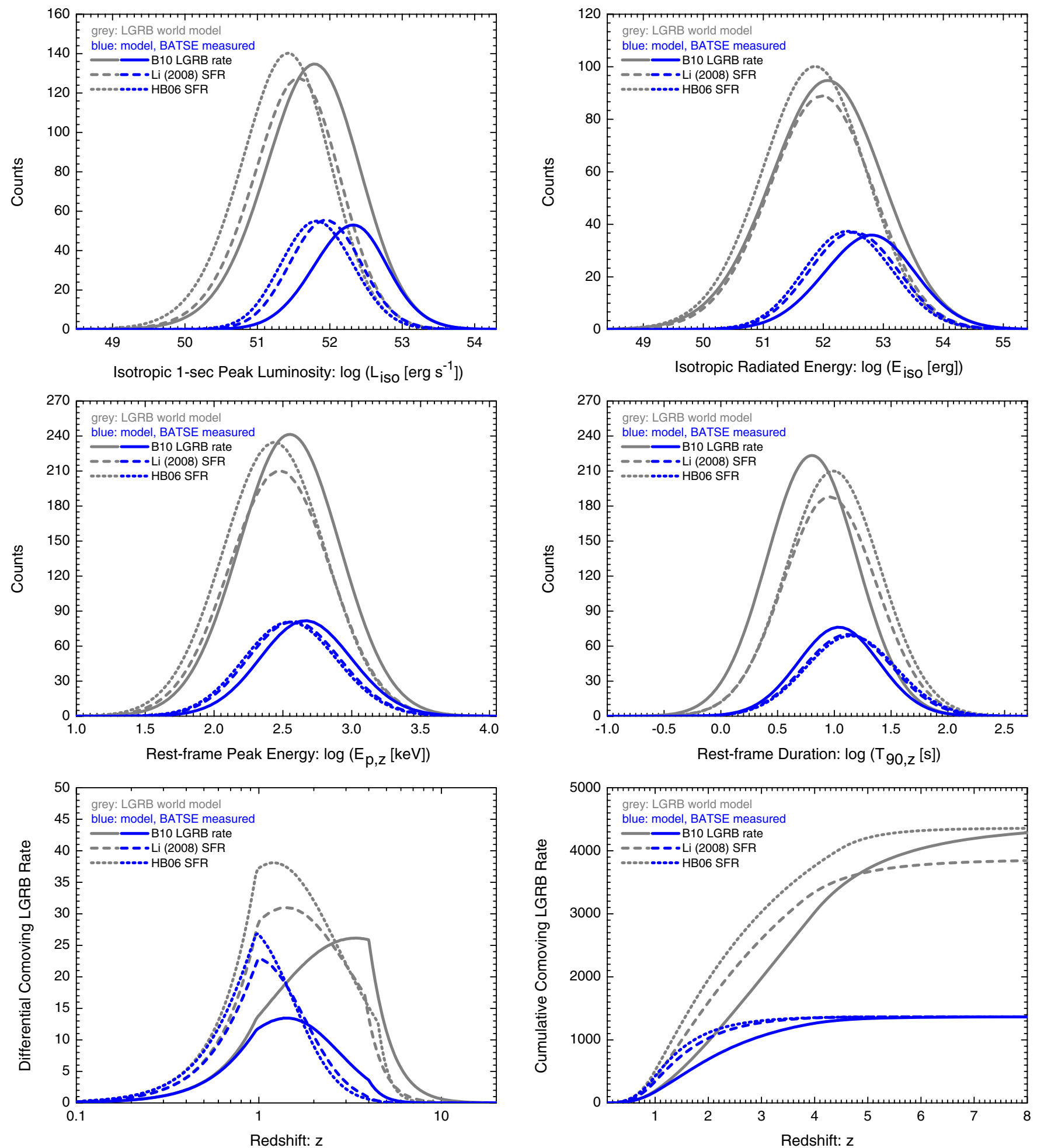

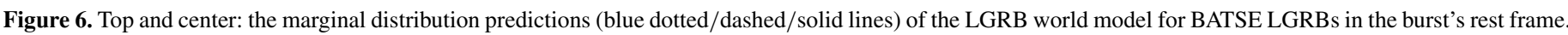

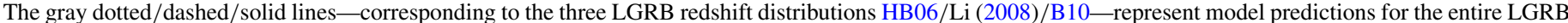

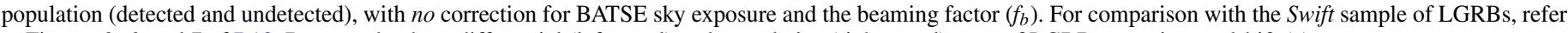
to Figures 2, 6, and 7 of B10. Bottom: the three differential (left panel) and cumulative (right panel) rates of LGRBs at a given redshift ( $z$ ).

(A color version of this figure is available in the online journal.)

Kouveliotou et al. 1993). Such a classification, however, has long been known to be ambiguous close to the cutoff set at $T_{90}=2 \mathrm{~s}$. It will be therefore useful to explore how accurate such classification is for the entire LGRB population (including non-triggered LGRBs). Figure 2 (center right plot) depicts the underlying population versus the 1366 BATSE LGRB observed durations $\left(T_{90}\right)$. As implied by the model, the shape of the $T_{90}$ distribution of LGRBs is not significantly affected by the triggering process of BATSE, since both BATSE and entire LGRB population distributions show a similar peak at $T_{90} \sim 30 \mathrm{~s}$. There is however a slight difference $(<0.1 \mathrm{dex})$ in the predicted observer-frame peak of LGRBs $T_{90}$ distribution, depending on 

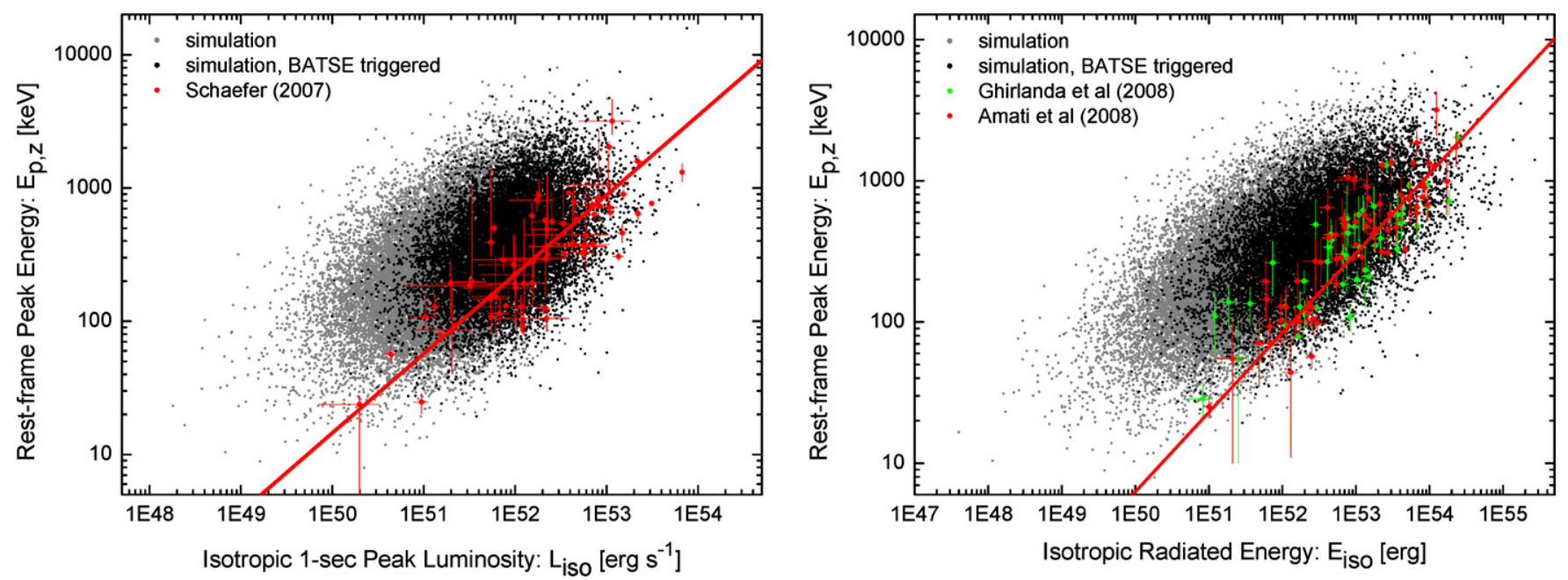

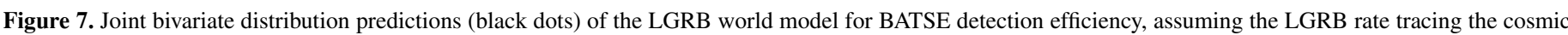

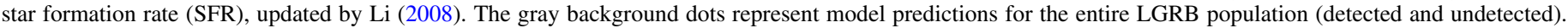

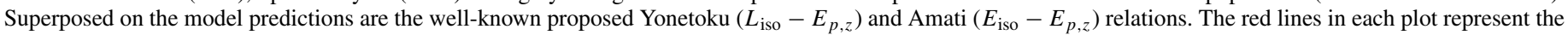

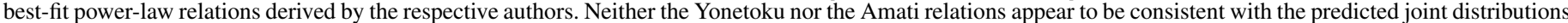

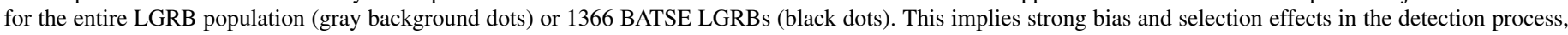
redshift determination, and spectral analysis of the LGRB samples that were used to construct the two relations.

(A color version of this figure is available in the online journal.)

the underlying LGRB redshift distribution assumed. The difference is magnified to $\sim 0.2 \mathrm{dex}$ in the rest-frame $\left(T_{90, z}\right)$ duration distribution of LGRBs, for the two extreme cases of HB06 and B10 redshift distributions. In general, a higher LGRB rate at high redshifts (as in the case of B10 redshift distribution) results in a shift to shorter durations in the duration distribution of LGRBs, in both the observer and the rest frames (Figure 6, center right plot).

Overall, the model predicts an average dynamic $3 \sigma$ range of observer-frame $\log \left(T_{90}(\mathrm{~s})\right) \in[1.47 \pm 1.32]$ corresponding to $T_{90}(\mathrm{~s}) \in[1.4,620]$ for LGRBs. This translates to an average dynamic $3 \sigma$ range of rest-frame $\log \left(T_{90, z}(\mathrm{~s})\right) \in[0.92 \pm 1.24]$ corresponding to $T_{90, z}(\mathrm{~s}) \in[0.47,145]$.

\subsection{Temporal and Spectral Correlations}

Ever since the launch of the Swift gamma-ray detector satellite, there has been a flurry of reports on the discovery of strong and significant correlations among the spectral parameters of LGRBs, most prominently among the rest-frame spectral peak energy and the total isotropic emission or peak luminosity of LGRBs (e.g., Amati et al. 2008; Ghirlanda et al. 2008). Despite the lack of measured redshifts for BATSE GRBs, signatures of such correlations had been found by earlier works in the BATSE era through careful analysis of observer-frame spectral properties of LGRBs (e.g., Lloyd et al. 2000). Nevertheless, the strength and significance of these correlations were undermined by analyzing larger samples of the BATSE catalog of GRBs (e.g., Nakar \& Piran 2005; Band \& Preece 2005; Shahmoradi \& Nemiroff 2009, 2011) or the Swift sample of GRBs (e.g., Butler et al. 2007, 2009; B10), all arguing that the sample of bursts used to construct the claimed spectral relations is representative of only bright-soft LGRBs. These arguments have been responded to by others (e.g., Ghirlanda et al. 2005, 2008; Nava et al. 2008; see Shahmoradi \& Nemiroff 2011 for a complete history of the debate).

A multivariate analysis of the BATSE LGRB data that carefully eliminates potential biases at the detection process can therefore greatly help us understand the strength and significance of the reported correlations. Figure 7 shows the predictions of the LGRB world model for the two widely discussed spectral correlations: the $L_{\text {iso }}-E_{p, z}$ (the Yonetoku) and $E_{\mathrm{iso}}-E_{p, z}$ (the Amati) relations. As indicated by the model, a large fraction of BATSE LGRBs (and a much larger fraction of the entire LGRB population) on the dim-hard regions of the plots appear to be underrepresented by the sample of LGRBs used for the construction of these relations. Nevertheless, given the three redshift distributions considered for the model, a relatively strong (Pearson's correlation coefficient $\rho_{E_{\mathrm{iso}}-E_{p, z}}=0.58 \pm$ $0.04)$ and highly significant $(>14 \sigma)$ correlation is predicted between the $E_{\text {iso }}$ and $E_{p, z}$ of LGRBs. The slope of the two relations suggested by the model also differs significantly from the original reports of the relations (Schaefer 2007; Ghirlanda et al. 2008). Generally, in regression modeling, it is assumed that there are two independent and dependent variables. In the case of the proposed relations, none of the variables is known to depend theoretically on the other. Therefore, due to the large statistically unexplained variances of the two LGRB variables, different regression methods, such as ordinary least squares, $\operatorname{OLS}(Y \mid X)$ and $\operatorname{OLS}(X \mid Y)$, result in entirely different slopes for the relations. The best-fit power-law relations and the conditional variances of the regress and given the regressor can be easily obtained from the parameters of the multivariate log-normal model in Table 2 (e.g., Section 2.11 in Kutner et al. 2004).

A partial correlation analysis of the two $L_{\mathrm{iso}}-E_{p, z}$ and $E_{\text {iso }}-E_{p, z}$ relations (Figure 8) reveals that the moderate correlation of the isotropic peak luminosity with the time-integrated peak energy is entirely due to the strong association of $L_{\text {iso }}$ with the total isotropic emission from the burst. As seen in the top left plot of Figure 8 there is indeed a negative correlation between the $L_{\mathrm{iso}}$ and $E_{p, z}$ of GRBs for a fixed isotropic emission $E_{\text {iso }}$ and burst duration $T_{90, z}$. Conversely, the model indicates a highly significant correlation of $E_{\text {iso }}$ with the time-integrated $E_{p, z}$, even after elimination of the effects of $L_{\text {iso }}$ and $T_{90, z}$ from the $E_{\text {iso }}-E_{p, z}$ relation. 

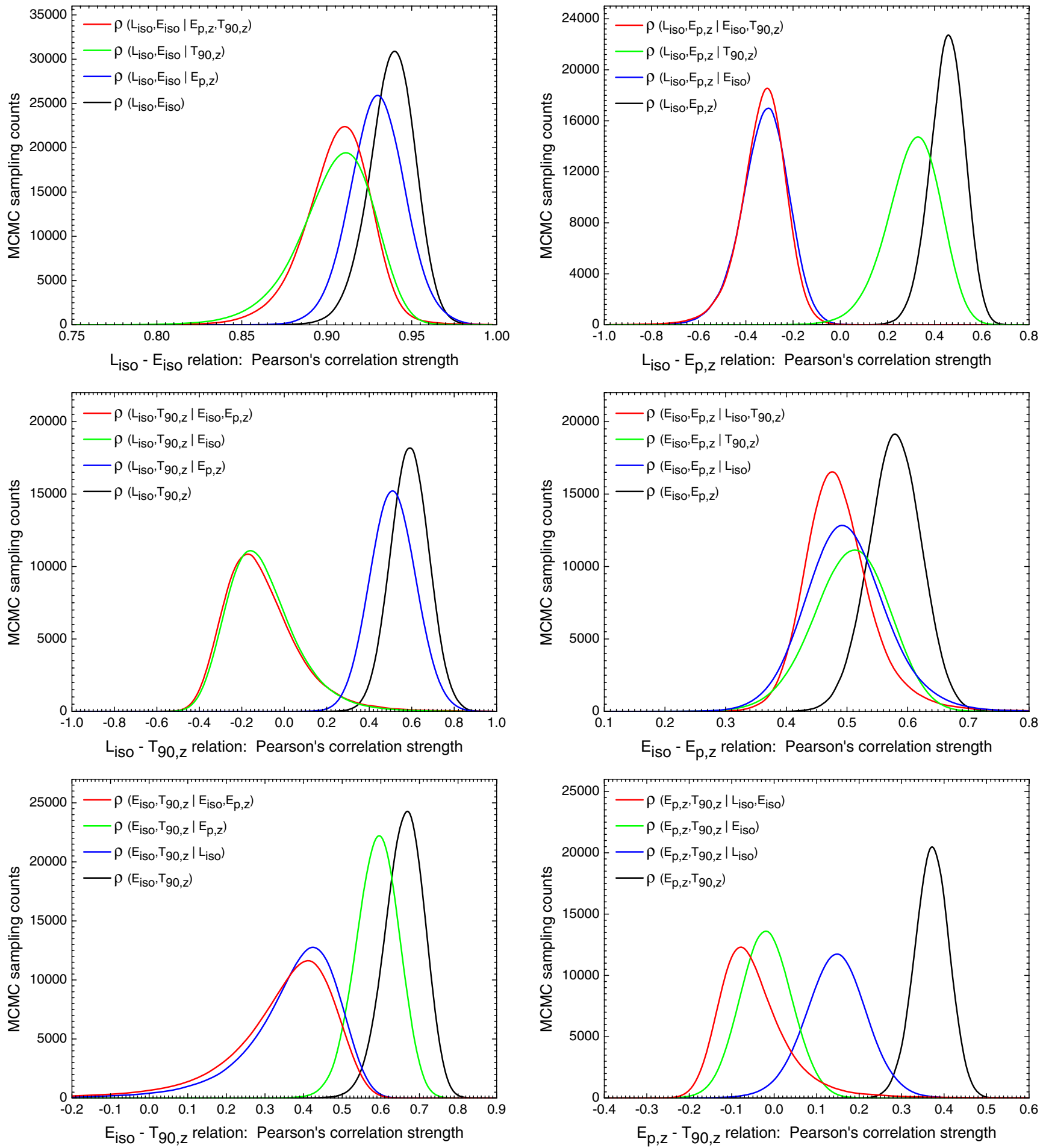

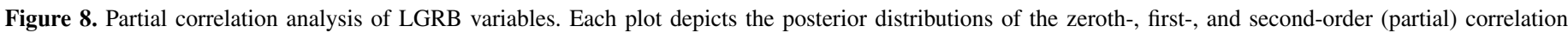

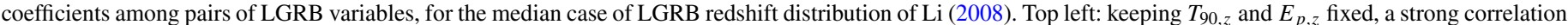

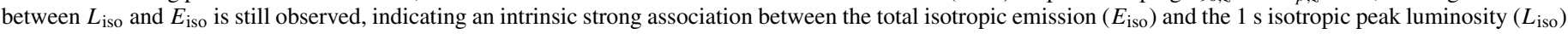

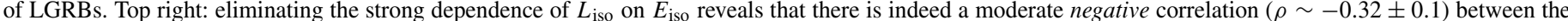

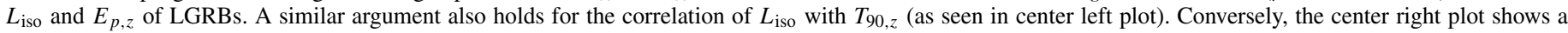

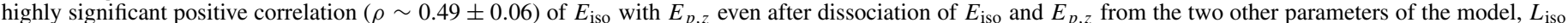

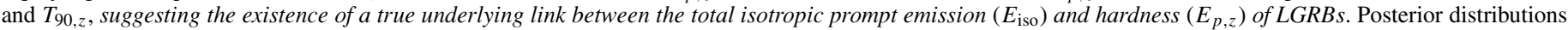
of the correlation coefficients in the case of the two other LGRB redshift distributions of HB06 and B10 also exhibit similar behavior.

(A color version of this figure is available in the online journal.)

As observed in Table 2, the model predicts positive correlation among all four LGRB variables. The isotropic peak luminosity $\left(L_{\text {iso }}\right)$ and the total isotropic emission $\left(E_{\text {iso }}\right)$ appear to be strong indicators of each other reciprocally. Surprisingly, it is also observed that the rest-frame duration $T_{90, z}$ of LGRBs strongly correlates with both $E_{\text {iso }}$ and $L_{\text {iso. }}$. The existence of a possible positive correlation between the isotropic emission and the duration of LGRBs has been implied by the analysis of Swift 

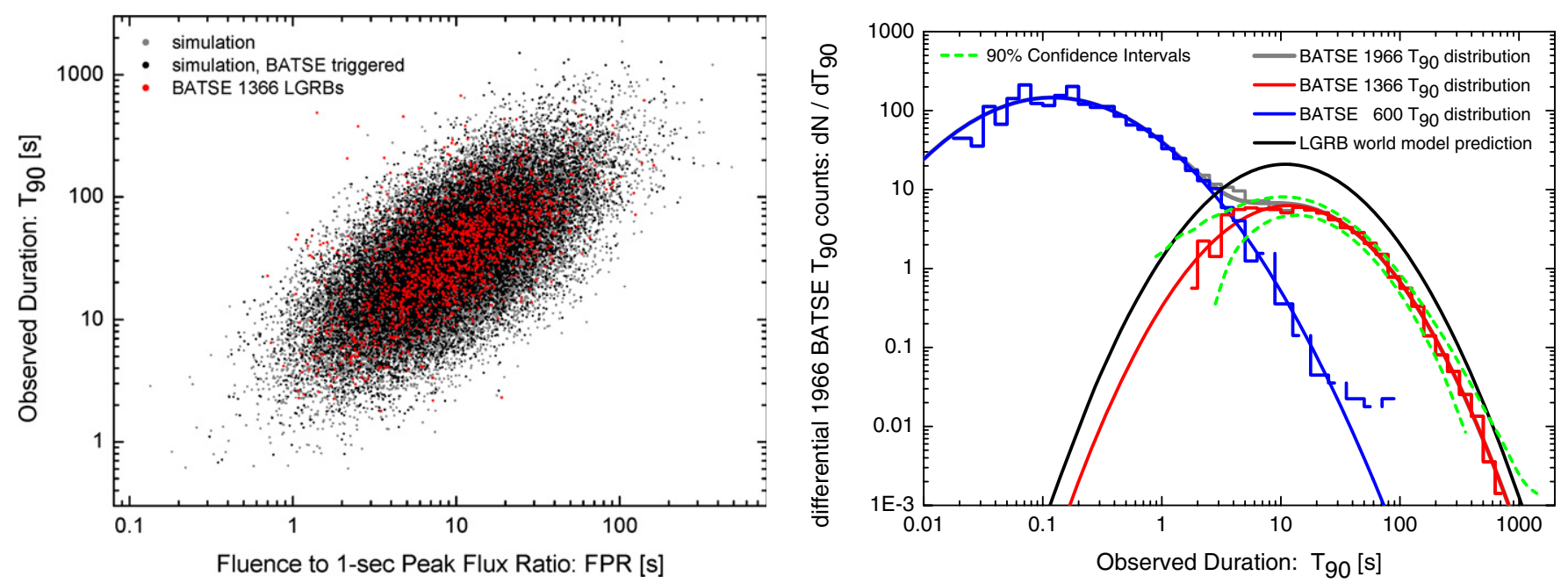

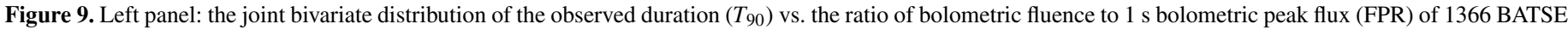

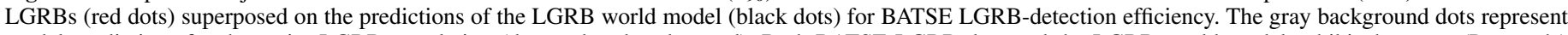

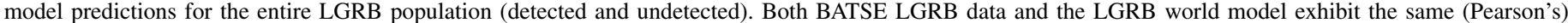

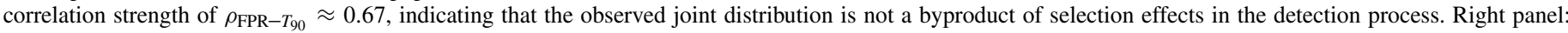

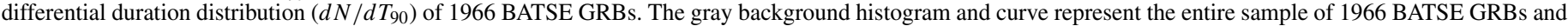

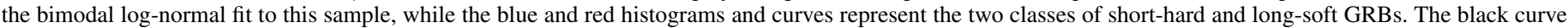

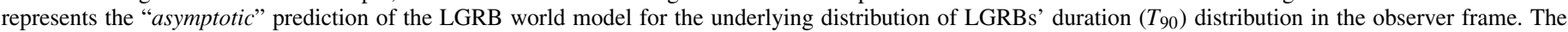

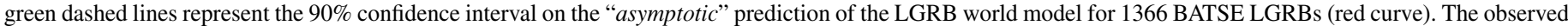

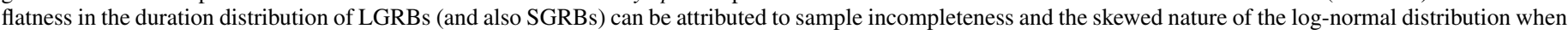

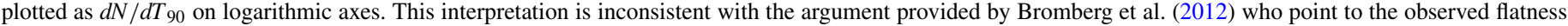
in $T_{90}$ distribution of BATSE GRBs as a direct evidence of the Collapsar model of LGRBs.

(A color version of this figure is available in the online journal.)

LGRBs (B10), though only weakly present there. Such correlations can be enlightening for the early studies of time-dilation signatures in BATSE GRBs. A positive duration-brightness correlation is also opposite to-but not necessarily in contradiction with - the negative duration-brightness correlation in pulses of individual GRBs (e.g., Fenimore et al. 1995; Nemiroff 2000; Ramirez-Ruiz \& Fenimore 2000). Combination of the two correlations implies that the number of pulses in individual LGRBs should be positively correlated with the peak luminosity (or equivalently, the total isotropic emission) of the bursts. This is indeed in qualitative agreement with the observed inequality relation between the isotropic peak luminosity and the number of pulses in Swift LGRBs (Figure 6 in Schaefer 2007). The strength of the correlations found encourages the search for the underlying physical mechanism that could give rise to these relations. This is, however, beyond the scope of this paper (cf. Rees \& Mészáros 2005; Ryde et al. 2006; Thompson et al. 2007; Giannios 2012; Dado \& Dar 2012 for example discussions).

It is also worth mentioning that the $T_{90}$ duration of BATSE LGRBs strongly correlates with the bolometric fluence to bolometric $1 \mathrm{~s}$ peak flux ratio (FPR), with Pearson's correlation coefficient $\rho_{\mathrm{FPR}-T 90} \approx 0.67$. A comparison of BATSE data with the predictions of the model for the bivariate distribution of FPR and $T_{90}$ is given in Figure 9 (left panel). Interestingly, the model predicts the same correlation strength of $\rho_{\mathrm{FPR}-T 90} \approx 0.67$ for the entire LGRB population, implying that the detection process does not bias the FPR- $T_{90}$ relation. Such strong correlation indicates an underlying intrinsic interrelation between the three variables: $P_{\mathrm{bol}}, S_{\mathrm{bol}}$, and $T_{90}$, also among their corresponding rest-frame counterparts. In fact, B10 use a variant of this trivariate correlation to define an effective peak flux in terms of fluence and duration, discarding the traditional definition of peak flux as the peak photon counts in $1 \mathrm{~s}$ time interval.

\section{SUMMARY AND CONCLUDING REMARKS}

The primary goal of the presented analysis was to model and constrain the LF, temporal and spectral correlations, and energetics of long-duration class of GRBs by exploiting the wealth of information that has been buried and untouched in the BATSE GRB catalog to this date. Below is a summary of steps taken to construct the LGRB world model, detailed in Section 2.

1. A sample of 1366 LGRBs is carefully selected by using the fuzzy C-means clustering algorithm based on temporal and spectral parameters and inspection of the individual light curves of BATSE-catalog GRBs (Figure 1; Section 2.1).

2. It is proposed that the BATSE LGRB data might be very well consistent with being drawn from a multivariate lognormal population of LGRBs in four rest-frame LGRB variables: the bolometric isotropic $1 \mathrm{~s}$ peak luminosity $\left(L_{\text {iso }}\right)$, the bolometric isotropic emission $\left(E_{\text {iso }}\right)$, the spectral peak energy $\left(E_{p, z}\right)$, and the duration $\left(T_{90, z}\right)$. Therefore, the observed joint distribution of the four LGRB variables: the bolometric $1 \mathrm{~s}$ peak flux $\left(P_{\mathrm{bol}}\right)$, the bolometric fluence $\left(S_{\mathrm{bol}}\right)$, the observed spectral peak energy $\left(E_{p}\right)$ and the observed duration $\left(T_{90}\right)$ results from the convolution of the restframe multivariate log-normal population with the cosmic rate (i.e., the redshift distribution) of LGRBs, truncated by the complex LGRB trigger threshold of BATSE LADs, as illustrated in Section 2.2, Equations (1)-(5). A prescription for modeling BATSE LAD detection efficiency is given in Appendix B.

3. The LGRB model (Equation (1)) is fit to BATSE data by maximizing the likelihood function of the model (Section 2.3 and Appendix C; Equation (C2)). In order to derive the best-fit parameters of the model and their corresponding uncertainties, an adaptive Metropolis-Hastings 
MCMC (AMH-MCMC) algorithm is set up to efficiently sample from the $16 \mathrm{D}$ likelihood function. The best-fit parameters are obtained for three LGRB cosmic rates: SFR of HB06, SFR of Li (2008), and the predicted LGRB redshift distribution of $\mathrm{B} 10$ which is consistent with the LGRB rate tracing cosmic metallicity with a cutoff $Z / Z_{\odot} \sim 0.2-0.5$.

4. To ensure the model provides adequate fit to observational data, multivariate GoF tests are presented (Section 2.4 and Figures 2-5).

Summarized below are the principal conclusions drawn from the analysis based on the proposed LGRB world model.

1. Energetics. It is expected that the peak brightness distribution of LGRBs has the effective range of $\log \left(P_{\mathrm{bol}}\left(\mathrm{erg} \mathrm{s}^{-1} \mathrm{~cm}^{-2}\right)\right) \in[-7.11 \pm 2.66]$ corresponding to $P_{\text {bol }}\left(\mathrm{erg} \mathrm{s}^{-1} \mathrm{~cm}^{-2}\right) \in\left[1.70 \times 10^{-10}, 3.58 \times 10^{-5}\right]$. This translates to a dynamic $3 \sigma$ range-in the rest frame-of $\log \left(L_{\text {iso }}\left(\mathrm{erg} \mathrm{s}^{-1}\right)\right) \in[51.53 \pm 1.99]$, corresponding to $L_{\text {iso }}\left(\mathrm{erg} \mathrm{s}^{-1}\right) \in\left[3.46 \times 10^{49}, 3.38 \times 10^{53}\right]$. In addition, a turnover is predicted in the differential $\log (N)-\log (P)$ diagram of LGRBs at $P_{50-300} \sim 0.1$ photons s ${ }^{-1} \mathrm{~cm}^{-2}$ in the BATSE nominal detection energy range (50-300 keV). This is consistent with and further extends the apparent flattening in the cumulative $\log (N)-\log (P)$ diagram of Swift LGRBs reported recently by $\mathrm{B} 10$.

As for the bolometric fluence and the total isotropic emission distributions, a range of $\log \left(S_{\mathrm{bol}}\left(\mathrm{erg} \mathrm{cm}^{-2}\right)\right) \in$ $[-6.16 \pm 3.01]$ corresponding to $S_{\mathrm{bol}}\left(\mathrm{erg} \mathrm{cm}^{-2}\right) \in$ $\left[6.82 \times 10^{-10}, 7.01 \times 10^{-4}\right]$ is indicated. This translates to an average dynamic $3 \sigma$ range-in the rest frame-of $\log \left(E_{\text {iso }}(\mathrm{erg})\right) \in[51.93 \pm 2.71]$ corresponding to $E_{\text {iso }}(\mathrm{erg}) \in\left[1.66 \times 10^{49}, 4.46 \times 10^{54}\right]$ (Sections 3.2 and 3.1; Table 2).

2. Durations and spectral peak energies. The rest-frame spectral peak energies $\left(E_{p, z}\right)$ of LGRBs are likely well described by a log-normal distribution with an average $3 \sigma$ range of $\log \left(E_{p, z}(\mathrm{keV})\right) \in[2.48 \pm 1.12]$ corresponding to $E_{p, z}(\mathrm{keV}) \in[23,4006]$ with peak LGRB rate at $E_{p, z} \sim 300(\mathrm{keV})$. This translates to an effective observerframe peak energy range of $\log \left(E_{p}(\mathrm{keV})\right) \in[1.93 \pm 1.22]$ corresponding to $E_{p}(\mathrm{keV}) \in[5,1427]$ with peak LGRB rate at $E_{p} \sim 85 \mathrm{keV}$. It is also observed that the observerframe $T_{90}$ duration of LGRBs peaks at $T_{90} \sim 30 \mathrm{~s}$ with a $3 \sigma$ range of $T_{90}(\mathrm{~s}) \in[1.4,620]$. This translates to an average $3 \sigma$ range of rest-frame $\log \left(T_{90, z}(\mathrm{~s})\right) \in[0.92 \pm 1.24]$ corresponding to $T_{90, z}(\mathrm{~s}) \in[0.47,145]$ with a peak rate at $T_{90, z} \sim 10$ s (Section 3.3; Table 2).

Recently, Bromberg et al. (2012) proposed the apparent flatness in the duration distribution of BATSE LGRBswhen plotted in the form of $d N / d T_{90}$ instead of $d N / d \log \left(T_{90}\right)$ - as the first direct evidence of the Collapsar model of LGRBs. The results of presented analysis are inconsistent with a flat $T_{90}$ distribution of LGRBs at short durations (Figure 2, center right panel and Figure 9, right panel). The observed flat $T_{90}$ distribution of LGRBs at short durations can be explained away in terms of the skewed nature of the log-normal distribution subject to sample incompleteness. It is therefore expected that a significantly larger sample of LGRBs that will be detected by future gamma-ray satellites will smear out the apparent flatness at the short tail of the duration distribution of LGRBs. A similar flat distribution is also observed for SGRBs at very short durations (Figure 9, right panel) which might be hard to reconcile with the Collapsar interpretation of the observed flatness in the LGRB $T_{90}$ distribution, proposed by Bromberg et al. (2012).

3. Temporal and spectral correlations. All four LGRB variables: $L_{\mathrm{iso}}, E_{\mathrm{iso}}, E_{p, z}$, and $T_{90, z}$ appear to be either moderately or strongly positively correlated with each other. In particular, a relatively strong and "broad" but highly significant correlation strength (Pearson's correlation coefficient $\left.\rho_{E_{\text {iso }}-E_{p, z}}=0.58 \pm 0.04\right)$ is predicted between $E_{\text {iso }}$ and $E_{p, z}$ of long-duration class of GRBs. Surprisingly, $T_{90, z}$ appears to evolve with $L_{\text {iso }}$ and $E_{\text {iso }}$ such that brighter bursts generally tend to have longer durations (Section 3.4; Table 2). This prediction of the model together with the previously reported negative correlation of the brightness and the duration of individual pulses in LGRBs (e.g., Fenimore et al. 1995; Nemiroff 2000; Ramirez-Ruiz \& Fenimore 2000) might possibly indicate that intrinsically brighter LGRBs contain, on average, a higher number of pulses.

There is a slight chance that a small fraction $(<50)$ of BATSE LGRBs were misclassified as SGRBs by the automated pattern recognition methods exploited in this analysis (see Figure 3, center right panel). If true, it will most likely affect (if significant at all) the constraints derived on the LF of LGRBs and the correlation of $E_{p, z}$ with $L_{\text {iso }}$.

4. Redshift distribution. The lack redshift information for the BATSE GRBs strongly limits the prediction power of the presented analysis for the cosmic rate of LGRBs. Nevertheless, based on the Markov chain sampling of the likelihood function for the three LGRB redshift distributions considered here (Section 2.2 and Figure 10), it is observed that BATSE data potentially, but not necessarily, favor an LGRB rate consistent with cosmic metallicity evolution with a cutoff $Z / Z_{\odot} \sim 0.2-0.5$ (cf. B10), with no luminosity-redshift evolution.

Assuming that LGRBs track SFR, only a tiny fraction (i.e., 2-3) of 1366 BATSE LGRBs are expected to have originated from high redshifts $(z \gtrsim 5)$. In the case of an LGRB rate tracing cosmic metallicity evolution (e.g., $\mathrm{B} 10$ ), the fraction increases by one order of magnitude to $\sim 2 \%$, corresponding to $\sim 27$ bursts out of 1366 BATSE LGRBs. For comparison, the expected fraction of Swift and EXIST LGRBs with $z \gtrsim 5$ are $\sim 6 \%$ and $\sim 7 \%$ (B10). The discrepancy is well explained by the fact that both Swift and EXIST are more sensitive to longsoft bursts - characteristic of high-redshift LGRBs - due to their lower gamma-ray trigger energy window, compared to BATSE DADs (cf. Gehrels et al. 2004; Band et al. 2008; Grindlay \& the EXIST Team 2009).

Although fitting is performed for the rest-frame variables, it is notable that the overall shape of the resulting observerframe distribution of the variables also resembles a multivariate log-normal (see Figures 2-7). In other words, the redshift convolution of the rest-frame population distribution approximately acts as a linear transformation from the rest frame of LGRBs to the observer frame. This is primarily due to the narrow redshift distribution of LGRBs - as compared to the width of the LGRB rest-frame temporal and spectral distributions-with almost $90 \%$ of the population originating from intermediate redshifts, $z \in[1,4.3]$. Balazs et al. (2003) provide an elegant discussion on the potential effects of redshift convolution on the observed distribution of LGRB durations and spectral parameters. 

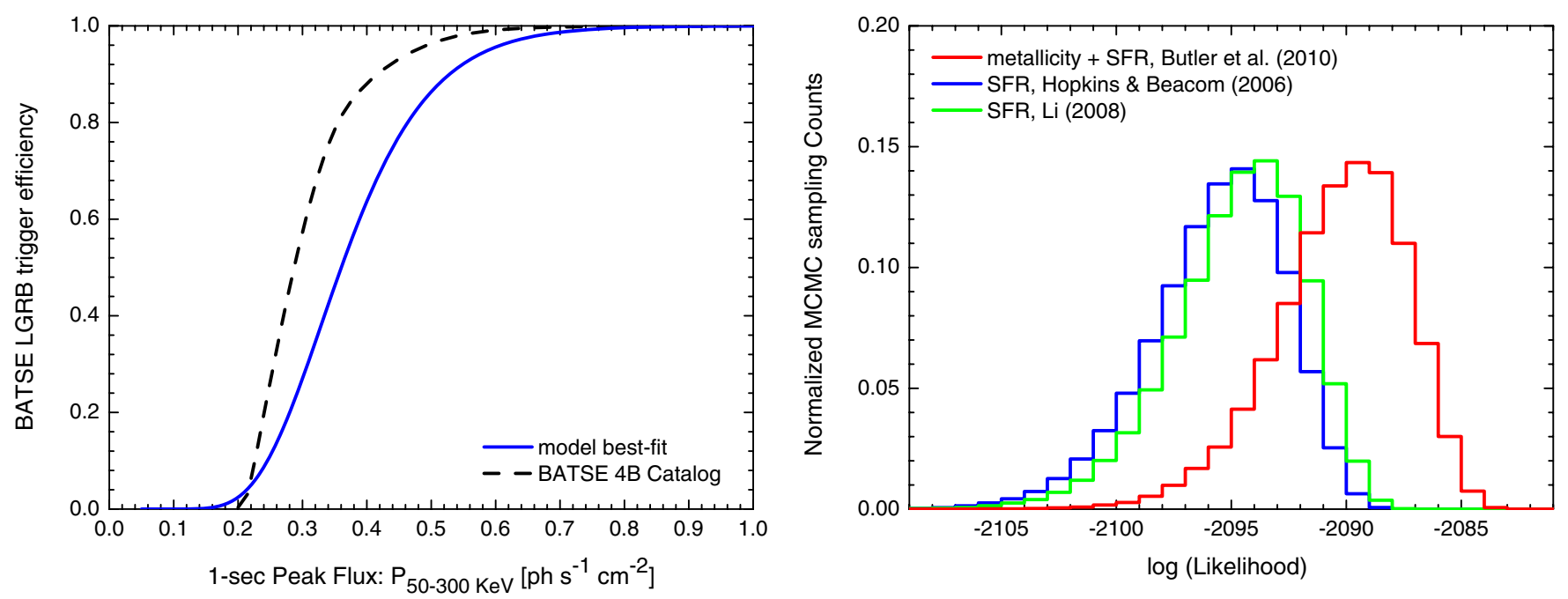

Figure 10. Left: a comparison of the model-predicted BATSE trigger efficiency of 1366 BATSE LGRBs with the nominal trigger efficiency estimates of BATSE 4B catalog. The discrepancy between the two curves is primarily due to different methodologies and GRB samples used to derive BATSE-catalog efficiency curve ${ }^{2}$ and the trigger efficiency of the LGRB world model. Right: the normalized sampling distribution of the likelihood function (Equation (C2)) from Markov chain for three LGRB redshift distributions (Section 2.2; Equation (4)). The fact that the redshift distribution of B10 generally results in larger likelihoods as compared to the two other can provide evidence— but does not necessitate in any way - that BATSE data favor an LGRB rate tracing cosmic metallicity evolution over SFR. A firm decision can only be made with complete knowledge of BATSE LGRB redshifts.

(A color version of this figure is available in the online journal.)

As implied by the model, there is no evidence for a significant population of bright-hard LGRBs that could have been missed in the BATSE catalog of GRBs. Conversely, a large population of low luminosity with moderate-to-low spectral peak energies seem to have gone undetected by BATSE LAD detectors. It should be emphasized that the apparent lack of very brightsoft LGRBs has a true physical origin according to the analysis presented here and is not an artifact of the detection process or spectral fitting models (e.g., the Band model, CPL or SBPL models) used by GRB researchers. Whether the X-Ray Flashes (XRF), X-ray-rich, and the sub-luminous GRBs (e.g., Strohmayer et al. 1998; Kippen et al. 2003; Tikhomirova et al. 2006) can be incorporated into a unified class of events described by a single model remains an open question in this work. At present, these events can be either considered as a separate class of cosmological events or as the soft-dim tail of the LGRB world model that have been mostly out of BATSE detection range and missed (see Figure 3 of Kippen et al. 2003 for a comparison with the predictions of the LGRB world model here in Figures 2-5). A definite answer to this question requires knowledge of the true rate of sub-luminous bursts and XRFs based on the observed rates of these events convolved with complex detection thresholds of different instruments used for observations.

B10 have presented an elaborate multivariate analysis of Swift LGRBs. While providing reasonable fit to Swift data, the model of B10 is primarily aimed at the discovery of the potential sub-luminous events that mostly go undetected by gammaray detectors. Such a model, capable of accounting for a large population of undetected sub-luminous bursts, is proposed at the cost of throwing away parts of information stored in the spectral parameters of LGRBs in the analysis of B10 (cf. Ghirlanda et al. 2012). Nevertheless, the presented analysis indicates that the apparent correlation of the isotropic peak luminosity $\left(L_{\text {iso }}\right)$ with the time-integrated spectral peak energy $\left(E_{p, z}\right)$ of LGRBs

\footnotetext{
2 http://www.batse.msfc.nasa.gov/batse/grb/catalog/4b/tables/ 4br_grossc.trig_sen
}

is peripheral to the more fundamental relation between the total isotropic emission $\left(E_{\mathrm{iso}}\right)$ and $E_{p, z}$, and that the relation can be created by defining an effective luminosity based on the two GRB variables $E_{\text {iso }}$ and duration (e.g., $T_{90, z}$ ) (Figures 8 and 9, left panel). In light of the analysis presented by Ghirlanda et al. (2012), it can be therefore suggested that a new definition of luminosity based on the $E_{\text {iso }}$ and $T_{90, z}$ of GRBs drawn from the LGRB world model of B10 will alleviate the apparent discrepancy between the observed $L_{\text {iso }}-E_{p, z}$ relation of LGRBs and the predicted relation from the LGRB model of B10. It is also expected that a better definition of peak luminosity that is not limited to a specific timescale in the observer frame of the bursts would result in an improvement in the correlations of the time-integrated spectral peak energy $\left(E_{p, z}\right)$ and the isotropic emission $\left(E_{\text {iso }}\right)$ with the luminosity variable $\left(L_{\text {iso }}\right)$. Given the above arguments, the presence of $L_{\text {iso }}$ as an independent variable in the LGRB world model-which was unfortunate due to the dependence of the BATSE trigger algorithm on the GRB's peak luminosity - might be viewed as overfitting and unnecessary.

The proposed multivariate log-normal model while requiring minimal free parameters compared to any other statistical model considered in GRB literature to date provides an accurate comprehensive description of the largest catalog of long-duration GRBs, serving as a powerful probe to explore the population properties of a large fraction of LGRBs that are missed in spectral analyses due to low-quality data or the lack of measured redshift, or simply go undetected due to the instrument's gamma-ray detector threshold. Data from future gamma-ray experiments will enable us to further confirm, improve, or invalidate predictions of the presented model.

The author owes special thanks to Robert J. Nemiroff (NASA GSFC Astrophysicist and professor of Physics at Michigan Tech University) for his generous support and help through numerous discussions over more than a year, from the early stages of developing the basic ideas behind this work to the core of the analysis and final steps of manuscript preparation. 
I thank William H. Press (professor of Computer Science and Integrative Biology at The University of Texas at Austin), Lars Koesterke (at Texas Advanced Computing Center), and Mehdi Mortazavi (at MTU) for helpful comments on some statistical and computational aspects of this work. I am very grateful to Swadesh Mahajan (professor at Institute for Fusion Studies) and Richard Hazeltine (professor and chair at the Department of Physics, The University of Texas at Austin) for their generous support in the final stages of preparing this manuscript. I also thank Giancarlo Ghirlanda (at INAF-Osservatorio Astronomico di Brera) for his timely feedback and comment on the GRB terminology used in this manuscript. Greatly appreciated were helpful comments and detailed criticisms from the anonymous referee of this manuscript.

This work would have not been accomplished without the vast time and effort spent by many scientists and engineers who designed, built, and launched the Compton Gamma-Ray Observatory and were involved in the analysis of GRB data from BATSE Large Area Detectors.

\section{APPENDIX A}

\section{GRB CLASSIFICATION}

It is well known that the traditional classification of GRBs based on a sharp cutoff in the observed duration $\left(T_{90}\right)$ distribution of GRBs-usually set at $T_{90}=2 \mathrm{~s}[50-300 \mathrm{keV}]-$ is insufficient and can be misleading close to the sharply defined border. The apparent long-soft-bright to short-hard-dim trend observed in the prompt-emission properties of BATSE GRBs (e.g., Figure 1, top panel; see also Figure 8 of Shahmoradi \& Nemiroff 2010) necessitates the use of a rigorous classification scheme based on all available spectral properties of GRBs, in addition to duration.

Despite a rich literature on the classification methodologies for GRBs (e.g., Hakkila et al. 2000a, 2003, and references therein), the choice of a classification method to separate the BATSE catalog of GRBs into two subgroups of long and short durations with minimal misclassifications remains a difficult task. This is primarily due to the significant overlap (or similarity) in all (or some) spectral and temporal properties of the two classes of GRBs, in addition to the heterogeneity of objective functions that might differ considerably from one classification algorithm to another.

Here, fuzzy (soft) clustering algorithms are preferred over hard clustering methods, since they provide a probability of the event belonging to each specific subgroup, in contrast to hard classifications that return only binary probabilities of either 0 or 1 . The choice of fuzzy algorithm greatly facilitates identification of bursts that might have been potentially misclassified (Section 2.1).

Investigation of different fuzzy algorithms available in the literature leads us to two prominent candidates: the SAND method of Rousseeuw et al. (1996) and the fuzzy C-means discussed by Dunn (1973) and Bezdek (1981). While fuzzy C-means is especially useful for cases where subgroups are known to be approximately symmetric, the SAND algorithm is superior to C-means for its lack of sensitivity to different subgroup sizes, orientations, and asymmetries. Nevertheless, the presence of a handful of SGRs in the BATSE catalog-with spectral properties comparable to that of LGRBs-results in relatively poor classification by the SAND method as compared to $\mathrm{C}$-means. Besides the choice of algorithm, the GRB variablesby which the classification is done-are selected such that the resulting relative sizes of the two SGRB and LGRB populations correspond to those found by Shahmoradi \& Nemiroff (2010) through a different approach that the author believes to be less prone to biases (cf. Figure 13 and Table 4 in Shahmoradi \& Nemiroff 2010).

\section{APPENDIX B}

\section{BATSE TRIGGER EFFICIENCY}

Before the LGRB world model of Equation (1) is fit to BATSE observational data, it is necessary to convolve the model with the BATSE trigger threshold (Equation (5)). The study of the BATSE detection efficiency is well documented in a series of articles by the BATSE team (e.g., Pendleton et al. 1995, 1998; Paciesas et al. 1999; Hakkila et al. 2003; cf. Shahmoradi \& Nemiroff 2011 for further discussion and references). Here, based on the observation that almost all 1366 BATSE LGRBs have durations of $T_{90}>1 \mathrm{~s}$, the primary trigger timescale for BATSE LGRBs is assumed to be $1024 \mathrm{~ms}$. This eliminates the relatively complex dependence of the detection probability $(\eta$ in Equation (5)) on the duration of the events. The probability of detection for an LGRB is then modeled by the cumulative density function (CDF) of log-normal distribution,

$$
\begin{aligned}
& \eta\left(\text { detection } \mid \mu_{\text {thresh }}, \sigma_{\text {thresh }}, L_{\text {iso }}, E_{p, z}, z\right) \\
& \quad=\frac{1}{2}+\frac{1}{2} \operatorname{erf}\left(\frac{\log \left(P\left(L_{\text {iso }}, E_{p, z}, z\right)\right)-\mu_{\text {thresh }}}{\sqrt{2} \sigma_{\text {thresh }}}\right),
\end{aligned}
$$

where $P\left(L_{\text {iso }}, E_{p, z}, z\right)$ is the $1 \mathrm{~s}$ peak photon flux in the BATSE nominal detection energy range: $50-300 \mathrm{keV}$, and $\mu_{\text {thresh }}$ and $\sigma_{\text {thresh }}$ are the detection threshold parameters to be determined by the model. The link between the $1 \mathrm{~s}$ peak photon flux $(P)$ and the LGRB rest-frame variables $\left(L_{\text {iso }}, E_{p, z}\right)$ and redshift $(z)$ is provided by fitting a smoothly broken power law known as the Band model (Band et al. 1993) to the differential photon spectra of LGRBs,

$$
\Phi(E) \propto \begin{cases}E^{\alpha} \exp \left(-(1+z)(2+\alpha) E / E_{p, z}\right) & E \leqslant\left[E_{p, z} /(1+z)\right] \\ & \times[(\alpha-\beta) /(2+\alpha)] \\ E^{\beta} & \text { otherwise, }\end{cases}
$$

such that

$$
P\left(L_{\mathrm{iso}}, E_{p, z}, z\right)=\frac{L_{\mathrm{iso}}}{4 \pi D_{L}^{2}(z)} \frac{\int_{50}^{300} \Phi d E}{\int_{0.1 /(1+z)}^{20000 /(1+z)} E \Phi d E} .
$$

It has been shown by B10 that fixing the high-energy and low-energy photon indices of the Band model (Equation (B2)) to the corresponding population average $\alpha=-1.1, \beta=$ -2.3 produces only a negligible error of $<0.05 \mathrm{dex}$ in the resulting flux estimates. Given the uncertainties in the BATSE LGRB variables, in particular $E_{p}$ estimates, such an assumption provides reasonable approximation for the calculation of the peak fluxes. A more accurate treatment, however, would be to include possible weak correlations that are observed between the Band model photon indices and the spectral peak energies of the bursts (cf. Shahmoradi \& Nemiroff $(2010,2011)$ for a discussion of the correlations and potential origins).

The goodness of the log-normal CDF assumption for BATSE detection efficiency can be checked by a comparison of the resulting model predictions with the 1366 BATSE LGRBs' distribution of peak fluxes (Figure 2). The best-fit model 
prediction of BATSE trigger efficiency for the long-duration class of GRBs is compared to the nominal trigger efficiency of the BATSE 4B catalog for the class of soft-long bursts in Figure 10 (left panel). Although the difference between the two curves is significant, it does not necessarily imply a contradiction, given the fact that different methodologies and GRB samples were used to derive the two efficiency curves. ${ }^{3}$

\section{APPENDIX C}

\section{LIKELIHOOD FUNCTION}

To obtain the joint posterior for the unknown parameters of the LGRB world model of Equation (1) given BATSE data, the likelihood function of the model must be, in principle, constructed by correctly accounting for uncertainties in observational data (e.g., Eddington 1913; Jeffreys 1938). In addition, it is known that astronomical surveys at low S/Ns close to survey threshold can be potentially biased (e.g., Hogg \& Turner 1998). A Bayesian multilevel methodology (e.g., Hobson et al. 2010) can incorporate the above corrections required to construct the likelihood function: under the assumption of normality for the uncertainties of LGRB variables in the BATSE catalog, each LGRB event-denoted by $\boldsymbol{O}_{i}$, standing for the $i$ th LGRB observation-has the likelihood $\mathcal{L}_{i}$ of having the true parameters $\boldsymbol{O}_{i} \equiv\left[P_{\mathrm{bol}, i}, S_{\mathrm{bol}, i}, E_{p, i}, T_{90, i}\right]$ that is described by a 4D Gaussian pdf:

$$
\mathcal{L}_{i}\left(\boldsymbol{O}_{i} \mid \boldsymbol{\mu}_{i, 0}, \Sigma_{i, 0}\right) \sim \mathcal{N}\left(\boldsymbol{O}_{i} \mid \boldsymbol{\mu}_{i, 0}, \Sigma_{i, 0}\right)
$$

where the location $\left(\mu_{i, 0}\right)$ and the scale $\left(\Sigma_{i, 0}\right)$ parameters of the pdf are to be determined by the model and individual event data from the BATSE GRB catalog. Given $\mathcal{L}_{i}$ and the LGRB world model (Equation (1)) convolved with BATSE trigger efficiency (Equation (5)), the full Poisson likelihood function can be written as

$$
\begin{aligned}
& \mathcal{L}(\text { Data } \mid \text { Model Parameters })=\mathcal{A}^{N} \exp \left(-\mathcal{A} \int_{\boldsymbol{O}_{\text {space }}} \mathcal{R}_{\text {obs }}(\boldsymbol{O}) d \boldsymbol{O}\right) \\
& \quad \times \prod_{i=1}^{N} \int_{\boldsymbol{O}_{\text {space }}} \mathcal{R}_{\text {cosmic }}\left(\boldsymbol{\mu}_{i, 0}, \Sigma_{i, 0}, z_{i} \mid \text { Model Parameters }\right) \mathcal{L}_{i}\left(\boldsymbol{O}_{i} \mid\right. \\
& \left.\quad \times \boldsymbol{\mu}_{i, 0}, \Sigma_{i, 0}\right) d \boldsymbol{O},
\end{aligned}
$$

where $N=1366$, and $\mathcal{A}$ is a factor that properly normalizes the cosmic and the observed rates $\left(\mathcal{R}_{\text {cosmic }}\right.$ and $\left.\mathcal{R}_{\text {obs }}\right)$. The term $\mathcal{R}_{\text {cosmic }}$ in Equation (C2) acts as a prior for $\mathcal{L}_{i}$. In the absence of knowledge of the prior (i.e., $\mathcal{R}_{\text {cosmic }}$, as in the case here), the empirical Bayes approach can provide an alternative solution, in which an ad hoc estimate of the model parameters based on the observed data (excluding uncertainties) serves as the prior for same data at the second level of analysis. Calculation of the normalization factor $\mathcal{A}$ involves an integration of the LGRB world model over the 5D space of LGRB variables and redshift, with a complex integration limit set by the BATSE trigger efficiency modeled in Equation (B1), as a function of $L_{\mathrm{iso}}, E_{p, z}$, and $z$. In addition, since almost no redshift information is available for BATSE catalog of GRBs, the probability for each LGRB has to be marginalized over redshift, for which a range of $z \in[0.1, \infty]$ is considered. These integrations make the maximization of the likelihood for all unknown parameters an extremely difficult task, perhaps

\footnotetext{
http://www.batse.msfc.nasa.gov/batse/grb/catalog/4b/4br_efficiency.html.
}

challenging current computational technologies. Moreover, it has been known that GRB fluences and durations are likely underestimated close to detection threshold due to the so-called fluence-duration bias (e.g., Hakkila et al. 2000b, 2003). Such bias makes the overestimation correction of the fluence and duration as prescribed by Hogg \& Turner (1998) unjustified before the fluence-duration bias effects are well quantified. The algorithms for calculating peak fluxes, however, appear to result in less biased measurements-even down to detector threshold-with negligible uncertainties (e.g., Stern et al. 2001). Given the above lines of reasoning and the computational limitations, the uncertainties on three BATSE LGRB variables, $P_{\text {bol }}, S_{\text {bol }}$, and $T_{90}$, are excluded from the likelihood function (Equation $(\mathrm{C} 2)$ ). The uncertainties on the $E_{p}$ estimates of BATSE LGRBs are, however, significant compared to the three former variables and must be incorporated in the calculations of the likelihood. Nevertheless, it was realized after likelihood maximization that the exclusion of the $E_{p}$ uncertainties-by fixing $E_{p}$ values to the "bisector" estimates of Shahmoradi \& Nemiroff $(2010)$ - results in only negligible $(\ll 1 \sigma)$ changes in the model's best-fit parameters. In general, the use of the bisector line of OLS regression lines (e.g., Isobe et al. 1990) for estimation purposes is unfavored due to lack of a maximum likelihood interpretation. The special case of BATSE LGRBs here, however, turns out to be an exception.

In addition to cosmological time-dilation correction, it is common practice to make an energy correction to the temporal variables of Swift GRBs (e.g., Gehrels et al. 2006; B10), such as $T_{90}$, when translating the variable from the observer frame to the rest frame. For BATSE LGRBs, this energy correction is likely negligible, given the fact that the $T_{90}$ durations are calculated based on the total photon counts in the BATSE LAD energy range (e.g., Kouveliotou et al. 1993; Fishman et al. 1994), 20-2000 keV, which can be practically considered as bolometric. Nevertheless, it is expected that such an energy correction, if needed, would slightly relax the strong correlation of the rest-frame duration $\left(T_{90, z}\right)$ with the total isotropic emission $\left(E_{\text {iso }}\right)$ and the peak isotropic luminosity $\left(L_{\text {iso }}\right)$.

The joint posterior distribution of the model parameters is obtained by maximizing the likelihood function of Equation (C2) convolved with a non-informative uniform prior on the location parameters and the standard choice of Jeffreys prior on the scale parameters (Jeffreys 1946). In order to efficiently sample from the $16 \mathrm{D}$ posterior density function, a variant of the MCMC methods known as AMH-MCMC is employed (e.g., Haario et al. 2001). The choice of an adaptive (versus classical) $\mathrm{MH}$ algorithm is very important, since the model parameters exhibit strong covariance (Table 3). To reduce the simulation runtime, all algorithms including AMH-MCMC are implemented in Fortran, which is by far the fastest and most efficient programming language for many intensive scientific calculations and number crunching (Loh 2010). In addition, the numerical integration in the definition of the luminosity distance (Equation (3))-encountered on the order of $\gtrsim 10^{9}$ times during the full MCMC sampling-is greatly simplified by the analytical approximation method of Wickramasinghe \& Ukwatta (2010). Due to the intrinsic sequential character of MCMC sampling methods, the parallelization of simulation algorithms (on either shared or distributed memory architecture machines) is impractical or at best inefficient for a single Markov chain. Nevertheless, to increase the MCMC sample size and more importantly, to ensure convergence to the global (versus local) extremum, the chain is initiated 
simultaneously at 10 random starting points in the parameter space on a 12-core desktop CPU. In general, convergence and good mixing occur within the first few thousands of iterations (burn-in period) given a suitable initial guess for the covariance matrix of the proposal distribution-here chosen to be multivariate Gaussian. The resulting mean and $1 \sigma$ standard deviations of the model parameters are tabulated in Table 2.

\section{APPENDIX D}

\section{LGRB MONTE CARLO UNIVERSE}

The prediction power and consistency of the presented LGRB world model—based on BATSE data-can be easily checked against observational data from current and future gamma-ray experiments, in particular the Fermi satellite. All it takes is to construct a Monte Carlo universe of LGRBs, based on the bestfit parameters of the LGRB world model in Table 2, and compare the outcome with observational data. Although straightforward, the steps for such simulation and comparison are summarized below.

1. A random redshift for each simulated LGRB is drawn from the redshift distribution of Equation (4) with parameters taken from Table 2. It is recommended to repeatedly randomly draw the set of model parameters from the full Markov chain samples ${ }^{4}$ instead of fixing the parameters to the mean values reported in Table 2.

2. The four LGRB variables: $L_{\text {iso }}, E_{\text {iso }}, E_{p, z}, T_{90, z}$ are randomly drawn from a 4D log-normal distribution with location $(\boldsymbol{\mu})$ and scale (i.e., the covariance matrix: $\Sigma$ ) parameters constructed from fitting results in Table 2 . This can be easily and quickly done by noting that a multivariate log-normal distribution is equivalent to a multivariate Gaussian distribution in the logarithmic space of the above variables,

$$
\log \boldsymbol{O} \equiv\left(\log \left(L_{\text {iso }}\right), \log \left(E_{\text {iso }}\right), \log \left(E_{p, z}\right), \log \left(T_{90, z}\right)\right),
$$

such that the 4D log-normal density function, $\mathcal{L N}$, of Equation (1) can be exactly replaced by a 4D Gaussian distribution,

$$
\begin{aligned}
\mathcal{N}(\log \boldsymbol{O} \mid \boldsymbol{\mu}, \Sigma) & =(2 \pi)^{-k / 2}|\Sigma|^{-1 / 2} \\
& \times \exp \left(-(\log \boldsymbol{O}-\boldsymbol{\mu})^{\prime} \Sigma^{-1}(\log \boldsymbol{O}-\boldsymbol{\mu}) / 2\right)
\end{aligned}
$$

for which the cosmic LGRB differential rate $\left(\mathcal{R}_{\text {cosmic }}\right)$ of Equation (1) will be

$$
\mathcal{R}_{\text {cosmic }}=\frac{d N}{d \log \left(L_{\mathrm{iso}}\right) d \log \left(E_{\mathrm{iso}}\right) d \log \left(E_{p, z}\right) d \log \left(T_{90, z}\right) d z},
$$

3. The above Monte Carlo universe of LGRBs can be then measured according to the instrument's detection efficiency (Section 2.2 and Equation (5)). For the case of BATSE LAD detectors, the trigger efficiency can be modeled according to the prescription in Appendix B.

\section{REFERENCES}

Amati, L., Frontera, F., Tavani, M., et al. 2002, A\&A, 390, 81 Amati, L., Guidorzi, C., Frontera, F., et al. 2008, MNRAS, 391, 577

\footnotetext{
4 Available at https://sites.google.com/site/amshportal/research/aca/ in-the-news/lgrb-world-model.
}

Atteia, J.-L., Barat, C., Hurley, K., et al. 1987, ApJS, 64, 305

Azzalini, A. 1985, Scand. J. Stat., 12, 171

Balazs, L. G., Bagoly, Z., Horvth, I., Mszros, A., \& Mszros, P. 2003, A\&A, 401, 129

Band, D., Matteson, J., Ford, L., et al. 1993, ApJ, 413, 281

Band, D. L. 2001, ApJ, 563, 582

Band, D. L. 2003, ApJ, 588, 945

Band, D. L. 2006, ApJ, 644, 378

Band, D. L., Grindlay, J. E., Hong, J., et al. 2008, ApJ, 673, 1225

Band, D. L., \& Preece, R. D. 2005, ApJ, 627, 319

Bezdek, J. C. 1981, Pattern Recognition with Fuzzy Objective Function Algorithms (Norwell, MA: Kluwer)

Bloom, J. S., Butler, N. R., \& Perley, D. A. 2008, in AIP Conf. Proc. 1000, Gamma-Ray Bursts 2007 Proc. Santa Fe Conf., ed. M. Galassi, D. Palmer, \& E. Fenimore (Melville, NY: AIP), 11

Boella, G., Butler, R. C., Perola, G. C., et al. 1997, A\&AS, 122, 299

Brainerd, J. J. 1997, ApJ, 487, 96

Briggs, M. S. 1993, ApJ, 407, 126

Bromberg, O., Nakar, E., Piran, T., \& Sari, R. 2012, ApJ, 749, 110

Burrows, D. N., Hill, J. E., Nousek, J. A., et al. 2005, SSRv, 120, 165

Butler, N. R., Bloom, J. S., \& Poznanski, D. 2010, ApJ, 711, 495 (B10)

Butler, N. R., Kocevski, D., \& Bloom, J. S. 2009, ApJ, 694, 76

Butler, N. R., Kocevski, D., Bloom, J. S., \& Curtis, J. L. 2007, ApJ, 671, 656

Campisi, M. A., Li, L.-X., \& Jakobsson, P. 2010, MNRAS, 407, 1972

Cochran, W. G. 1954, Biometrics, 10, 417

Dado, S., \& Dar, A. 2012, ApJ, 749, 100

Dermer, C. D. 1992, PhRvL, 68, 1799

Dezalay, J.-P., Atteia, J.-L., Barat, C., et al. 1997, ApJL, 490, L17

Dunn, J. C. 1973, J. Cybern., 3, 32

Eddington, A. S. 1913, MNRAS, 73, 359

Fenimore, E. E., Conner, J. P., Epstein, R. I., et al. 1988, ApJL, 335, L71

Fenimore, E. E., Epstein, R. I., Ho, C., et al. 1993, Natur, 366, 40

Fenimore, E. E., 't Zand, J. J. M., Norris, J. P., Bonnell, J. T., \& Nemiroff, R. J. 1995, ApJL, 448, L101

Fisher, R. A. 1924, J. R. Stat. Soc., 87, 442

Fishman, G. J., Meegan, C. A., Wilson, R. B., et al. 1994, ApJS, 92, 229

Gehrels, N., Chincarini, G., Giommi, P., et al. 2004, ApJ, 611, 1005

Gehrels, N., Norris, J. P., Barthelmy, S. D., et al. 2006, Natur, 444, 1044

Gehrels, N., Ramirez-Ruiz, E., \& Fox, D. B. 2009, ARA\&A, 47, 567

Ghirlanda, G., Ghisellini, G., Firmani, C., Celotti, A., \& Bosnjak, Z. 2005, MNRAS, 360, L45

Ghirlanda, G., Ghisellini, G., \& Lazzati, D. 2004, ApJ, 616, 331

Ghirlanda, G., Ghisellini, G., Nava, L., et al. 2012, MNRAS, 422, 2553

Ghirlanda, G., Nava, L., Ghisellini, G., Firmani, C., \& Cabrera, J. I. 2008, MNRAS, 387,319

Giannios, D. 2012, MNRAS, 422, 3092

Grindlay, J., \& the EXIST Team 2009, in AIP Conf. Proc. 1133, Gamma-Ray Burst: Sixth Huntsville Symp., ed. C. Meegan, C. Kouveliotou, \& N. Gehrels (Melville, NY: AIP), 18

Guetta, D., Piran, T., \& Waxman, E. 2005, ApJ, 619, 412

Haario, H., Saksman, E., \& Tamminen, J. 2001, Bernoulli, 7, 223

Hakkila, J., Giblin, T. W., Roiger, R. J., et al. 2003, ApJ, 582, 320

Hakkila, J., Haglin, D. J., Roiger, R. J., et al. 2000a, in AIP Conf. Proc. 526, Gamma-Ray Bursts: Fifth Huntsville Symp., ed. R. M. Kippen, R. S. Mallozzi, \& G. J. Fishman (Melville, NY: AIP), 33

Hakkila, J., Meegan, C. A., Pendleton, G. N., et al. 2000b, in AIP Conf. Proc. 526, Gamma-Ray Bursts: Fifth Huntsville Symp., ed. R. M. Kippen, R. S. Mallozzi, \& G. J. Fishman (Melville, NY: AIP), 48

Hakkila, J., Pendleton, G. N., Meegan, C. A., et al. 2003, in AIP Conf. Proc. 662, Gamma-Ray Burst and Afterglow Astronomy 2001, ed. G. R. Ricker \& R. K. Vanderspek (Melville, NY: AIP), 176

Hobson, M. P., Jaffe, A. H., Liddle, A. R., Mukeherjee, P., \& Parkinson, D. 2010, Bayesian Methods in Cosmology (Cambridge: Cambridge Univ. Press)

Hogg, D. W., \& Turner, E. L. 1998, PASP, 110, 727

Hopkins, A. M., \& Beacom, J. F. 2006, ApJ, 651, 142 (HB06)

Isobe, T., Feigelson, E. D., Akritas, M. G., \& Babu, G. J. 1990, ApJ, 364, 104

Jarosik, N., Bennett, C. L., Dunkley, L., et al. 2011, ApJS, 192, 14

Jeffreys, H. 1938, MNRAS, 98, 190

Jeffreys, H. 1946, RSPSA, 186, 453

Justel, A., Pea, D., \& Zamar, R. 1997, Stat. Probab. Lett., 35, 251259

Kippen, R. M., Woods, P. M., Heise, J., et al. 2003, in AIP Conf. Proc. 662, Gamma-Ray Burst and Afterglow Astronomy 2001, ed. G. R. Ricker \& R. K. Vanderspek (Melville, NY: AIP), 244

Klebesadel, R. W., Strong, I. B., \& Olson, R. A. 1973, ApJL, 182, L85

Kolmogoroff, A. 1941, Ann. Math. Stat., 12, 461

Kommers, J. M., Lewin, W. H. G., Kouveliotou, C., et al. 2000, ApJ, 533, 696 Kouveliotou, C., Meegan, C. A., Fishman, G. J., et al. 1993, ApJL, 413, L101 
Kumar, P., \& Piran, T. 2000, ApJ, 535, 152

Kutner, M. H., Neter, J., Nachtsheim, C. J., \& Li, W. 2004, Applied Linear Statistical Models (5th ed.; New York: McGraw-Hill)

Levesque, E. M. 2012, in IAU Symp. 279, The Death of Massive Stars, Supernovae and Gamma-Ray Bursts, ed. P. W. A. Roming, N. Kawai, \& E. Pian (Cambridge: Cambridge Univ. Press), 167

Levesque, E. M., Berger, E., Kewley, L. J., \& Bagley, M. M. 2010a, AJ, 139,694

Levesque, E. M., Kewley, L. J., Graham, J. F., \& Fruchter, A. S. 2010b, ApJL, $712, \mathrm{~L} 26$

Levesque, E. M., Soderberg, A. M., Foley, R. J., et al. 2010c, ApJL, 709, L26

Li, L.-X. 2007, MNRAS, 374, L20

Li, L.-X. 2008, MNRAS, 388, 1487

Lloyd, N. M., Petrosian, V., \& Mallozzi, R. S. 2000, ApJ, 534, 227

Loh, E. 2010, Queue, 8, 30:30

Mallozzi, R. S., Paciesas, W. S., Pendleton, G. N., et al. 1995, ApJ, 454, 597

Mazets, E. P., \& Golenetskii, S. V. 1981, Ap\&SS, 75, 47

Meegan, C. A., Fishman, G. J., Wilson, R. B., et al. 1992, Natur, 355, 143

Metzger, M. R., Djorgovski, S. G., Kulkarni, S. R., et al. 1997, Natur, 387,878

Nakar, E. 2007, PhR, 442, 166

Nakar, E., \& Piran, T. 2005, MNRAS, 360, L73

Nava, L., Ghirlanda, G., Ghisellini, G., \& Firmani, C. 2008, MNRAS, 391, 639

Nemiroff, R. J. 2000, ApJ, 544, 805

Nemiroff, R. J., Norris, J. P., Bonnell, J. T., et al. 1994, ApJL, 435, L133

Nishimura, J. 1988, in Physics of Neutron Stars and Black Holes, ed. Y. Tanaka (Tokyo: Universal Academy Press), 413

Norris, J. P., Bonnell, J. T., Kazanas, D., et al. 2005, ApJ, 627, 324

Paciesas, W. S., Meegan, C. A., Pendleton, G. N., et al. 1999, ApJS, 122, 465

Paczynski, B. 1986, ApJL, 308, L43

Peacock, J. A. 1983, MNRAS, 202, 615

Pendleton, G. N., Hakkila, J., \& Meegan, C. A. 1998, in AIP Conf. Proc. 428 Gamma-Ray Bursts: Fourth Huntsville Symp., ed. C. A. Meegan, R. D. Preece, \& T. M. Koshut (Melville, NY: AIP), 899

Pendleton, G. N., Paciesas, W. S., Mallozzi, R. S., et al. 1995, NIMPA, 364, 567

Petrosian, V. 1993, ApJL, 402, L33

Petrosian, V., \& Lee, T. T. 1996, ApJL, 467, L29

Petrosian, V., Lloyd, N., \& Lee, A. 1999, in ASP Conf. Ser. 190, GammaRay Bursts: The First Three Minutes, ed. J. Poutanen \& R. Svensson (San Francisco, CA: ASP), 235

Porciani, C., \& Madau, P. 2001, ApJ, 548, 522

Press, S. 1972, J. Multivariate Anal., 2, 444
Press, W. H., Teukolsky, S. A., Vetterling, W. T., \& Flannery, B. P. 1992, Numerical Recipes in FORTRAN. The Art of Scientific Computing (Cambridge: Cambridge Univ. Press)

Racusin, J. L., Oates, S. R., Schady, P., et al. 2011, ApJ, 738, 138

Ramirez-Ruiz, E., \& Fenimore, E. E. 2000, ApJ, 539, 712

Rees, M. J., \& Mészáros, P. 2005, ApJ, 628, 847

Reichart, D. E., \& Lamb, D. Q. 2001, in AIP Conf. Proc., 586, Relativistic Astrophysics: 20th Texas Symposium, ed. D. E. Reichart \& D. Q. Lamb (Melville, NY: AIP), 599

Rousseeuw, P., Kaufman, L., \& Trauwaert, E. 1996, Comput. Stat. Data Anal., 23, 135

Ryde, F., Bjrnsson, C.-I., Kaneko, Y., et al. 2006, ApJ, 652, 1400

Salvaterra, R., Campana, S., Vergani, S. D., et al. 2012, ApJ, 749, 68

Salvaterra, R., \& Chincarini, G. 2007, ApJL, 656, L49

Salvaterra, R., Guidorzi, C., Campana, S., Chincarini, G., \& Tagliaferri, G. 2009, MNRAS, 396, 299

Schaefer, B. E. 2007, ApJ, 660, 16

Schmidt, M. 1999, ApJL, 523, L117

Schmidt, M. 2001, ApJ, 552, 36

Schmidt, M. 2009, ApJ, 700, 633

Sethi, S., \& Bhargavi, S. G. 2001, A\&A, 376, 10

Shahmoradi, A., \& Nemiroff, R. 2009, in AIP Conf. Proc., 1133, Gamma-ray Burst: Sixth Huntsville Symposium, ed. C. Meegan, C. Kouveliotou, \& N Gehrels (Melville, NY: AIP), 425

Shahmoradi, A., \& Nemiroff, R. J. 2010, MNRAS, 407, 2075

Shahmoradi, A., \& Nemiroff, R. J. 2011, MNRAS, 411, 1843

Smirnov, N. 1948, Ann. Math. Stat., 19, 279

Stanek, K. Z., Gnedin, O. Y., Beacom, J. F., et al. 2006, AcA, 56, 333

Stern, B. E., Atteia, J.-L., \& Hurley, K. 2002, ApJ, 578, 304

Stern, B. E., Tikhomirova, Y., Kompaneets, D., Svensson, R., \& Poutanen, J. 2001, ApJ, 563, 80

Strohmayer, T. E., Fenimore, E. E., Murakami, T., \& Yoshida, A. 1998, ApJ, 500,873

Thompson, C., Mészáros, P., \& Rees, M. J. 2007, ApJ, 666, 1012

Tikhomirova, Y., Stern, B. E., Kozyreva, A., \& Poutanen, J. 2006, MNRAS, 367,1473

Wanderman, D., \& Piran, T. 2010, MNRAS, 406, 1944

Wang, F. Y., \& Dai, Z. G. 2011, ApJL, 727, L34

Wickramasinghe, T., \& Ukwatta, T. N. 2010, MNRAS, 406, 548

Woosley, S. E. 1993, ApJ, 405, 273

Woosley, S. E., \& Heger, A. 2006, ApJ, 637, 914

Yonetoku, D., Murakami, T., Nakamura, T., et al. 2004, ApJ, 609, 935

Zhang, B., Zhang, B.-B., Liang, E.-W., et al. 2007, ApJL, 655, L25 